\title{
Multiblock variable influence on orthogonal projections (MB-VIOP) for enhanced interpretation of total, global, local and unique variations in OnPLS models
}

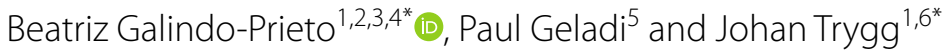

\section{${ }^{*}$ Correspondence: beg4004@med.cornell.edu; johan.trygg@umu.se ${ }^{1}$ Department of Chemistry, Computational Life Science Cluster (CLiC), Umeå University, Umeå, Sweden Full list of author information is available at the end of the article}

\begin{abstract}
Background: For multivariate data analysis involving only two input matrices (e.g., $X$ and $Y$ ), the previously published methods for variable influence on projection (e.g., $V_{\text {VIP }}$ OPLS or VIP O2PLS $_{\text {) }}$ are widely used for variable selection purposes, including (i) variable importance assessment, (ii) dimensionality reduction of big data and (iii) interpretation enhancement of PLS, OPLS and O2PLS models. For multiblock analysis, the OnPLS models find relationships among multiple data matrices (more than two blocks) by calculating latent variables; however, a method for improving the interpretation of these latent variables (model components) by assessing the importance of the input variables was not available up to now.

Results: A method for variable selection in multiblock analysis, called multiblock variable influence on orthogonal projections (MB-VIOP) is explained in this paper. MB-VIOP is a model based variable selection method that uses the data matrices, the scores and the normalized loadings of an OnPLS model in order to sort the input variables of more than two data matrices according to their importance for both simplification and interpretation of the total multiblock model, and also of the unique, local and global model components separately. MB-VIOP has been tested using three datasets: a synthetic four-block dataset, a real three-block omics dataset related to plant sciences, and a real six-block dataset related to the food industry.

Conclusions: We provide evidence for the usefulness and reliability of MB-VIOP by means of three examples (one synthetic and two real-world cases). MB-VIOP assesses in a trustable and efficient way the importance of both isolated and ranges of variables in any type of data. MB-VIOP connects the input variables of different data matrices according to their relevance for the interpretation of each latent variable, yielding enhanced interpretability for each OnPLS model component. Besides, MB-VIOP can deal with strong overlapping of types of variation, as well as with many data blocks with very different dimensionality. The ability of MB-VIOP for generating dimensionality reduced models with high interpretability makes this method ideal for big data mining, multi-omics data integration and any study that requires exploration and interpretation of large streams of data.
\end{abstract} author(s) and the source, provide a link to the Creative Commons licence, and indicate if changes were made. The images or other third party material in this article are included in the article's Creative Commons licence, unless indicated otherwise in a credit line to the material. If material is not included in the article's Creative Commons licence and your intended use is not permitted by statutory regulation or exceeds the permitted use, you will need to obtain permission directly from the copyright holder. To view a copy of this licence, visit http:// creativecommons.org/licenses/by/4.0/. The Creative Commons Public Domain Dedication waiver (http://creativecommons.org/publi cdomain/zero/1.0/) applies to the data made available in this article, unless otherwise stated in a credit line to the data. 
Keywords: Multiblock variable selection, OnPLS, VIP, MB-VIOP, Variable importance in multiblock regression, Latent variable interpretation, Variable influence on projection, Feature selection

\section{Background}

Multivariate data analysis can involve thousands of input (manifest) variables in just one data block. These variables may contain latent information that can help (i) to extract inferences and explain phenomena and relationships that might not be obvious from the experimental results obtained in the laboratory, (ii) to get a more meaningful and visual interpretation of the data, (iii) to optimize processes in both industry and research environments, and (iv) to understand the holistic pattern in complex biological systems where different parts interact by underlying connections. Compared to the analysis of a single dataset, the analysis of a large number of datasets (blocks) implies that the number of variables and their underlying inter-connections grow very much indeed; at this point, reducing the number of variables involved in the multiblock data analysis becomes a meaningful and much needed strategy.

Interest in multiblock approaches has risen in psychology [1-3], chemistry [4-7], biology $[8,9]$ and sensory science $[10,11]$, among other; an interest mainly motivated by the goal of extracting the maximum useful information from two or more datasets interrelated among themselves. Early multiblock methods based on projections and latent variables, e.g. partial least squares (PLS) [12, 13], allowed the analysis of a limited number (usually two or three) of data matrices, but without taking full advantage of how the data blocks were connected. Two commonly used multiblock approaches based on principal components were consensus principal component analysis (CPCA) [14, 15] and hierarchical principal component analysis (HPCA) [16], whose algorithms are very similar, differing only in the normalization steps [5]. For PLS applied to multiblock analysis, it is worth mentioning hierarchical partial least squares (HPLS) [14] and multiblock partial least squares (MBPLS) [17], which are similar but with two main differences: (i) the normalization is done on different model parameters, and (ii) the regression of the $\mathbf{Y}$-block is done on different matrices [5]. Some interesting applications of multiblock-PLS were reported by Wise and Gallagher in 1996 [18], and a better understanding of the underlying patterns in latent models was attempted by Kourti et al. [4] using multiblock multiway PLS for analyzing batch polymerization processes in 1995. Although many different multiblock methods based in different criteria and principles can be found in the literature (e.g. regularized generalized canonical correlation analysis, RGCCA [19]), this paper will mainly keep its scope inside methods based on partial least squares regression [20-30], such as sparse partial least squares presented by Le Cao et al. [31] (and further implemented by Rohart et al. [32]). Multiblock methods based on orthogonal projections have received interest within life-sciences provided the model structure it can decompose the data blocks into; two examples of this are the multi-omics factor analysis (MOFA) presented by Argelaguet et al. in 2018 [33] and the N-block orthogonal projections to latent structures (OnPLS) method presented by Löfstedt and Trygg in 2011 [34]. The latter can be used to provide some input parameters for improved model interpretation using MB-VIOP. From a methodology perspective, OnPLS provides means to take full advantage of the shared and unique variations of more than two data blocks. 
Examples of alternative methods with different objective functions include JIVE (joint and individual variation explained) [35], GSVD (generalized singular value decomposition) [36], and msPLS (multiset sparse partial least squares path modelling) [37].

The numerous variable selection methods for multivariate analysis of one data matrix [38-47] cannot handle the complexity and the underlying patterns of a large number of datasets; therefore, data integration and multiblock variable selection methods are needed. An important consideration is to be aware of the multiset structure since the integration of multiple datasets can be performed in different ways, and different methods may have specific requirements on this aspect. For instance, OnPLS followed by MB-VIOP has a similar integration framework than the N-integration of block sparse PLS requiring the same number of samples $(\mathrm{N})$ for all data matrices, whilst mint sparse PLS has a K-integration (also called P-integration in the literature) framework which requires the same number of variables $(K)$ instead of the same number of samples [32]. Besides, some methods are more suitable for improving model interpretability, whilst other are more suitable for improving predictability; hereby, the importance of selecting the appropriate variable selection method according to the purpose of the data analysis, an example of this was shown by comparing the obtained root mean square error of prediction (RMSEP) using two different variable selection methods on the Marzipan dataset in Galindo-Prieto et al. [48]. The fact that variable influence on projection (VIP) approaches for OPLS (VIP ${ }_{\text {OPLS }}$ ) [39], O2PLS (VIP O2PLS $_{148}$ [4 and OnPLS (MB-VIOP) base their calculations on the product between the normalized loadings ( $\mathrm{p}$ ) and the sum of squares of $\mathrm{X}$ and $\mathrm{Y}$ leads to an enhanced model interpretability that other methods cannot achieve. However, if the aim of the analysis is to achieve enhanced model predictability, other methods such as sparse PLS [31] (that uses the Q2 parameter as criterion to choose the number of model components, and the root means square error of prediction criterion for evaluation of the predictive power of each $Y$ variable between the original non penalized PLS models and the sparse PLS model) may be more suitable. We include a comparison for unsupervised multiblock variable selection using the sparse PLS method for multiblock cases (block-sPLS) [32] and MB-VIOP in the Results and Discussion section.

In addition, variable selection aiming to enhance the interpretation of latent variables containing uncorrelated (orthogonal) variation can be challenging. An example of an approach able to deal with multiple datasets is the sparse generalized canonical correlation analysis (SGCCA) for variable selection that combines RGCCA with the L1-penalty [49]; however, to deal also with orthogonalization in an analysis of multiple datasets,

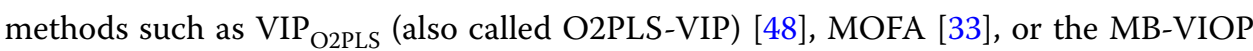
explained here are more suitable options. We include a comparison for unsupervised integrated feature selection between MOFA and MB-VIOP in the Results and Discussion section.

It is worth mentioning that for one PLS component, loadings or weights can be used for determining which variables are more influential [50], but this has limited use. There is a need for a diagnostic giving the described variable influence in a PLS model, or any of its derived orthogonal versions, using more than 1 component. All VIP diagnostics are constructed for that purpose. 
A multiblock variable selection method called multiblock variable influence on orthogonal projections (MB-VIOP) for OnPLS models was developed as part of previous thesis work [51] and is now published and explained in this paper. The mathemat-

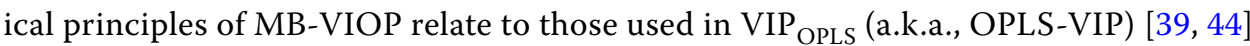

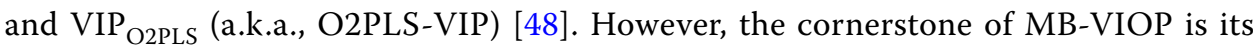
inter-block connectivity with emphasis on the variable influence, making MB-VIOP substantially different (i) from its two predecessors VIP ${ }_{\text {OPLS }}$ and VIP O2PLS $_{\text {in terms of }}$ connectivity, and also (ii) from OnPLS regression [34] since the normalized OnPLS p loadings cannot provide by themselves a reliable and precise variable importance assessment while this is easily achieved by MB-VIOP by taking these normalized loadings as starting point for the variable importance assessment (as it will be shown in the synthetic example). MB-VIOP allows the selection of the most important variables for enhanced interpretation of OnPLS models when three or more data blocks are simultaneously modelled. It is worth mentioning that MB-VIOP is also applicable to O2PLS ${ }^{\circledR}$ models that involve only two data blocks. Furthermore, MB-VIOP provides four MB-VIOP profiles (total, global, local and unique) to help answer questions such as:

a. Total MB-VIOP profile: Which are the variables that are more relevant for the interpretation of the whole model? Which variables could be eliminated from the model in order to improve it?

b. Global MB-VIOP profile: Which variables help to interpret the variation that is common to all the data blocks involved in the model?

c. Local MB-VIOP profile: Which variables are important to interpret the variation that is common to some of (but not all) the blocks? And how do these variables connect among the data blocks to explain the information shared by them (i.e., the variation related to the same component or latent variable)?

d. Unique MB-VIOP profile: Which are the variables that contain unique information that can be only found in one specific data block? And which inferences related to the data can be elucidated from the selected variables in the unique MB-VIOP profiles?

The MB-VIOP algorithm has been tested by using three multiblock datasets, (i) a simulated four-block dataset called SD16_235GLU, (ii) a real three-block omics dataset here called Hybrid Aspen, and (iii) a real six-block industrial dataset called Marzipan. The three datasets are described in detail in sections "Synthetic dataset (four blocks)"-"Metabolomics, proteomics and transcriptomics data of hybrid aspen (three blocks)".

\section{Results and discussion}

The results and the discussion aim to validate the multiblock variable influence on orthogonal projections (MB-VIOP) method for its application in OnPLS models (extended interpretations related to biology or spectroscopy are out of the scope of this paper). Thus, an OnPLS model followed by an MB-VIOP variable selection will 
be performed in all multiblock analyses. The input variables will be sorted according to their importance for the entire multiblock model (i.e., the total variation), but also for each model component separately (i.e., the unique, the local and the global variations). Figure 1 shows the different types of variation present in a generic OnPLS model.

\section{Description of the OnPLS models}

For the synthetic four-block SD16_235GLU data, an OnPLS model was built in MATLAB. The OnPLS algorithm found two global components (in black and blue in Fig. 2), three local components (in cyan, orange and green in Fig. 2), and three unique components (in pink color in Fig. 2); which points to a conservative, but well conducted, modelling by the OnPLS algorithm. Only two unique components included in the design of the synthetic data were not found; i.e., one unique component in block $\mathbf{D}_{\mathbf{1}}$ (which represented a $14.3 \%$ of the variation of $\mathbf{D}_{1}$ ) and one unique component in block $\mathbf{D}_{4}$ (which contained a $20 \%$ of the variation of $\mathbf{D}_{4}$ ). The rest of the variation was extracted by the model (see Table 1); the percentage of total variation explained by the model was $85.8 \%$ for $\mathbf{D}_{1}, 100 \%$ for $\mathbf{D}_{2}, 100 \%$ for $\mathbf{D}_{3}$ and $80 \%$ for $\mathbf{D}_{4}$.

For the Marzipan data, the six data matrices were used to generate an OnPLS model, which yielded two global components and two unique components (the percentages of explained variation per component and per block are shown in Table 2). The model was able to explain almost all variation; more specifically, a $96.2 \%$ of total variation for the NIRS1 block, a $93.8 \%$ for the NIRS2 block, a $95.8 \%$ for the INFRAPROVER block,

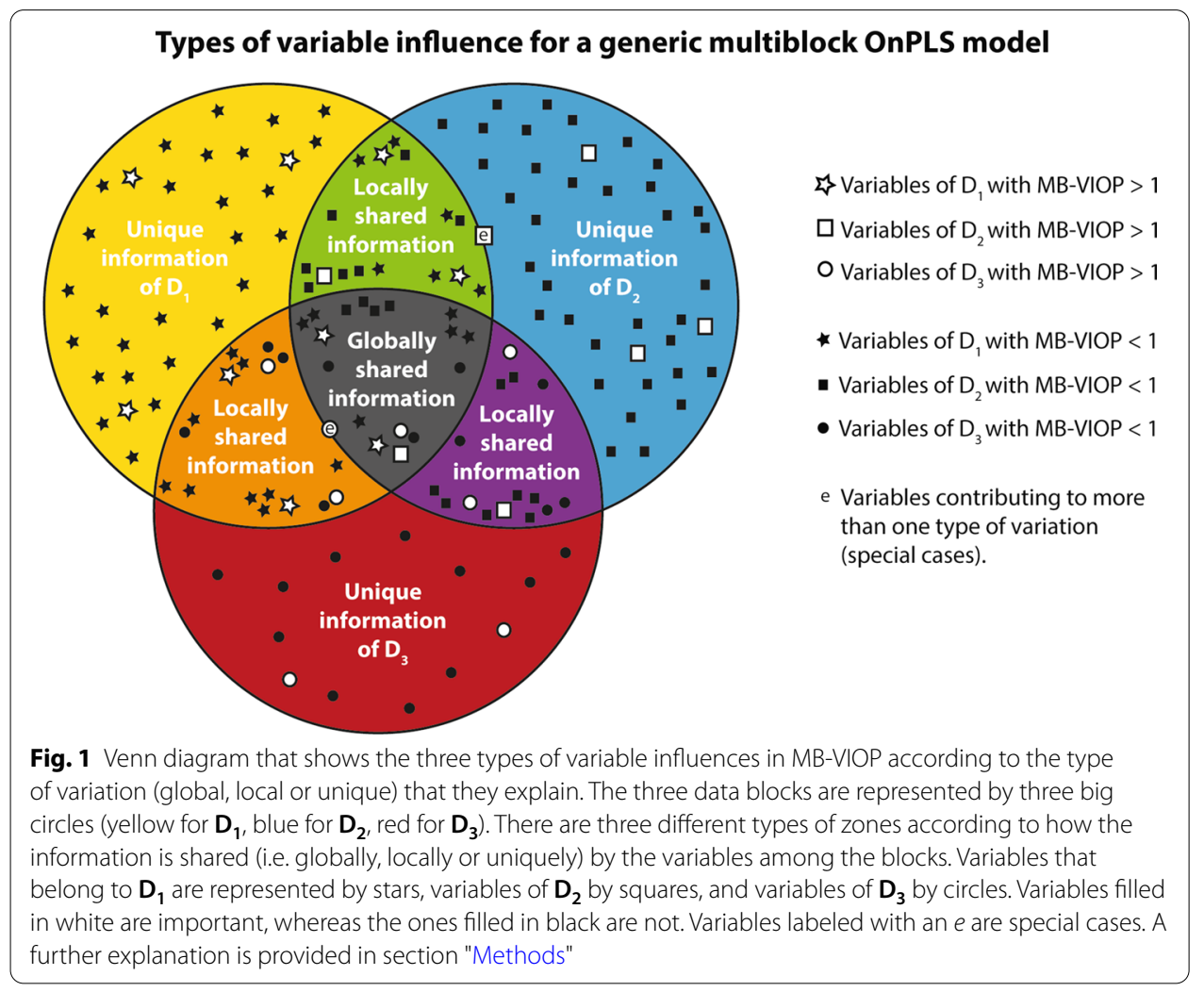




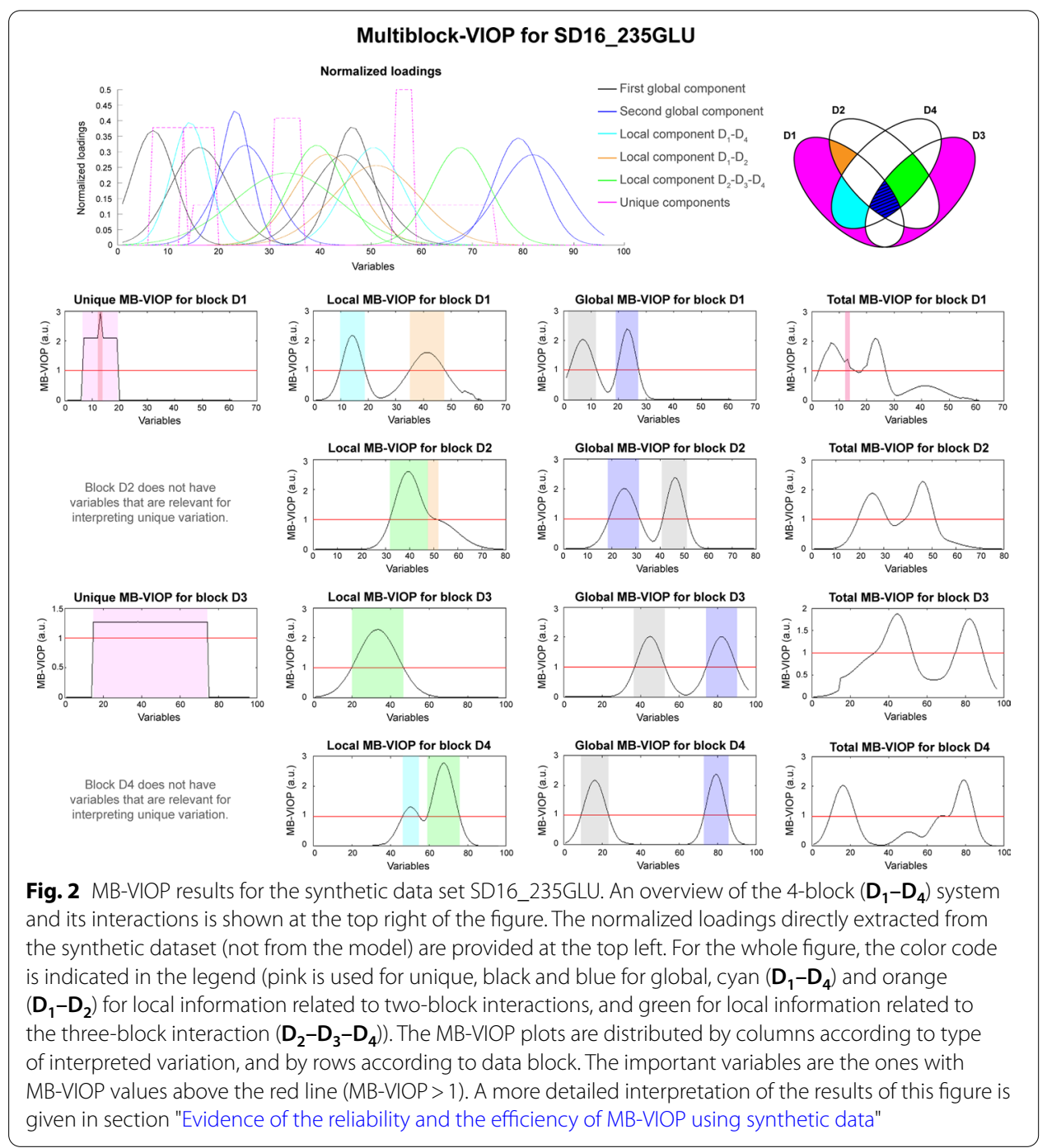

Table 1 Values of explained variation per data block (D1-D4) and per component for the OnPLS model of the SD16_235GLU dataset

\begin{tabular}{|c|c|c|c|c|c|c|c|c|}
\hline \multicolumn{9}{|c|}{ SD16_235GLU MODEL } \\
\hline \multicolumn{9}{|c|}{ Percentage of explained variation per data block and per component } \\
\hline Data block & $a_{\mathrm{g} 1}$ & $a_{\mathrm{g} 2}$ & $a_{11}$ & $a_{12}$ & $a_{13}$ & $a_{\mathrm{u} 1}$ & $a_{\mathrm{u} 2}$ & $a_{\mathrm{u} 3}$ \\
\hline D1 & 14.3 & 14.3 & 14.3 & 14.3 & & 14.3 & 14.3 & \\
\hline D2 & 25.0 & 25.0 & & 25.0 & 25.0 & & & \\
\hline D3 & 25.0 & 25.0 & & & 25.0 & & & 25.0 \\
\hline D4 & 20.0 & 20.0 & 20.0 & & 20.0 & & & \\
\hline
\end{tabular}

Values are given as percentages (\%), $a$ stands for component, $g$ for global, I for local, and $u$ for unique

a $97.0 \%$ for the BOMEM block, a $99.9 \%$ for the INFRATECH block and a $75.5 \%$ for the IR block. Since all blocks are related to NIR/IR spectroscopy, it is not surprising that the OnPLS algorithm found two global components. The Marzipan data mostly has 
Table 2 Values of explained variation per data block and per component for the OnPLS model of the Marzipan dataset

\begin{tabular}{|c|c|c|c|c|}
\hline \multicolumn{5}{|l|}{ Marzipan model } \\
\hline \multicolumn{5}{|c|}{ Percentage of explained variation per data block and per model component } \\
\hline Data block & $a_{g 1}$ & $a_{\mathrm{g} 2}$ & $\mathrm{a}_{\mathrm{u} 1}$ & $a_{\mathrm{u} 2}$ \\
\hline NIRS1 & 76.3 & 11.1 & 8.8 & \\
\hline NIRS2 & 90.5 & 3.3 & & \\
\hline INFRAPROVER & 84.7 & 11.1 & & \\
\hline BOMEM & 94.2 & 2.8 & & \\
\hline INFRATECH & 99.2 & 0.7 & & \\
\hline IR & 41.5 & 26.9 & & 7.1 \\
\hline
\end{tabular}

Values are given as percentages (\%), $a$ stands for component, $g$ for global, and $u$ for unique

Table 3 Values of explained variation per data block and per component for the OnPLS model of the Hybrid Aspen dataset

\begin{tabular}{|c|c|c|c|c|c|c|c|c|}
\hline \multicolumn{9}{|c|}{ Hybrid aspen model } \\
\hline \multicolumn{9}{|c|}{ Percentage of explained variation per data block and per component } \\
\hline Data block & $a_{g 1}$ & $a_{\mathrm{g} 2}$ & $a_{\mathrm{g} 3}$ & $a_{g 4}$ & $a_{11}$ & $a_{12}$ & $\mathrm{a}_{\mathrm{u} 1}$ & $\mathrm{a}_{\mathrm{u} 2}$ \\
\hline Transcriptomics & 11.9 & 30.9 & 12.0 & 2.4 & 4.4 & 5.3 & 8.1 & \\
\hline Proteomics & 17.8 & 14.4 & 10.6 & 4.0 & & 8.2 & & \\
\hline Metabolomics & 12.3 & 14.2 & 7.8 & 6.1 & 5.7 & & & 12.3 \\
\hline
\end{tabular}

Values are given as percentages (\%), a stands for component, $g$ for global, I for local, and $u$ for unique

predictive (joint) variation, which is absolutely dominant over the orthogonal (unique) variation [48].

For the Hybrid Aspen data, an OnPLS model was built obtaining four global components, two local components (one shared between the transcript and the metabolite data, and another shared between the transcript and the protein data), and two unique components (one for the transcriptomics block, and another for the metabolomics block). The OnPLS model explained $75.0 \%$ of the total variation for the transcriptomics data block (14,738 variables), 55.0\% for the proteomics data block (3132 variables), and $58.3 \%$ for the metabolomics data block (281 variables). The decomposition of explained variation for the different types of variation is shown in Table 3.

\section{Evidence of the reliability and the efficiency of MB-VIOP using synthetic data}

For the variation contained in the local component that $\mathbf{D}_{\mathbf{1}}$ shares with $\mathbf{D}_{4}, \mathrm{MB}$-VIOP selected as relevant variables 10-18, represented as a peak marked in cyan in the local MB-VIOP plot for $\mathbf{D}_{\mathbf{1}}$ (Fig. 2); in the same local MB-VIOP plot, variables 35-47 (marked in orange) were considered important for explaining the variation that $\mathbf{D}_{1}$ shares with $\mathbf{D}_{\mathbf{2}}$. The unique MB-VIOP plot for $\mathbf{D}_{\mathbf{1}}$ pointed at variables 7-19 as the important ones for explaining the unique variation of $\mathbf{D}_{\mathbf{1}}$; interestingly, variable 13 stood out from the rest of variables.

By comparing the MB-VIOP variable importance results to the normalized loadings (Fig. 2), it can be seen that the MB-VIOP method is very reliable finding the exact 
variables that are important for the different types of variation of $\mathbf{D}_{\mathbf{1}}$; furthermore, MBVIOP assesses the correct proportion of importance for each variable, which cannot be achieved by the normalized loadings plot. Hence, looking at variable 13 in the normalized loadings plot, it can be seen that this variable was related to the two unique components of $\mathbf{D}_{\mathbf{1}}$ (explaining $28.6 \%$ of variation), whereas the other variables (7-12 and 14-19) linked to the unique variation of $\mathbf{D}_{1}$ were only related to one of the unique components (explaining only $14.3 \%$ of the variation); however, the normalized loading plot did not highlight such an important variable (no. 13) in any way. Auspiciously, MBVIOP highlighted the importance of variable 13 (marked in dark pink color in Fig. 2) as an intense peak standing out from the crowd; this variable was also depicted in the total MB-VIOP plot for $\mathbf{D}_{\mathbf{1}}$. Therefore, the total and the unique MB-VIOP plots for $\mathbf{D}_{\mathbf{1}}$ evidence the efficiency of MB-VIOP algorithm to not lose track of any variable, even if it is a lonely variable.

The MB-VIOP results obtained for block $\mathbf{D}_{2}$ are encouraging, since, even with a high overlapping of the normalized loadings (profiles), the MB-VIOP algorithm identified the variables that were relevant for each type of variation (see Fig. 2).

For block $\mathbf{D}_{3}$, the variables considered important in the global MB-VIOP plot (Fig. 2) contributed to explain a $50 \%$ of the total variation of the OnPLS model, whilst the variables related to explain other types of variation did not overpass the $25 \%$; therefore, the variables related to the information globally shared by all the data matrices were selected as the most important ones for the whole model, leaving out the variables related to information that was local or unique. The unique variation of $\mathbf{D}_{3}$ ( $25 \%$ of the total variation) was explained by the large range of variables 15-74. For an overview assessment of the variable importance, the total MB-VIOP plot pointed at variables 33-52 and 75-89 as the most relevant ones. Interestingly, the total MB-VIOP plot emphasizes the efficiency of MB-VIOP giving the proportionally fair importance to the variables according to the amount of information that they help to explain in the OnPLS model; the absence of the large amount of variables which were relevant for the unique variation (i.e., variables 15-74 of $\mathrm{D}_{3}$ ) enlightened another achievement of the MB-VIOP algorithm: it does not matter if there is an outsize number of variables that are important for a specific type of variation, in case that their importance for interpreting/explaining variation in the whole model is not significant enough, they will not be considered relevant variables in the total MB-VIOP plot. The latter fact demonstrates that MB-VIOP properly sorts the variables according to their importance for explaining a specific type of variation.

\section{Enhancement of the interpretability in an OnPLS model for the Marzipan case by using MB-VIOP}

The MB-VIOP results (see Fig. 3) obtained for the OnPLS model generated using the Marzipan dataset (previously described in section "Description of the OnPLS models") helped to better interpret the pattern of information overlapping between the six data matrices (that would be a painstaking task if it was done by using the normalized loadings provided in Fig. 3). There is not significant amount of local variation in the Marzipan dataset, which explains the fact that no important variables for explaining local variation were selected by MB-VIOP. In addition, due to the extreme dominance of the joint variation over the unique variation, the MB-VIOP results for the global latent 
Multiblock-VIOP for Marzipan
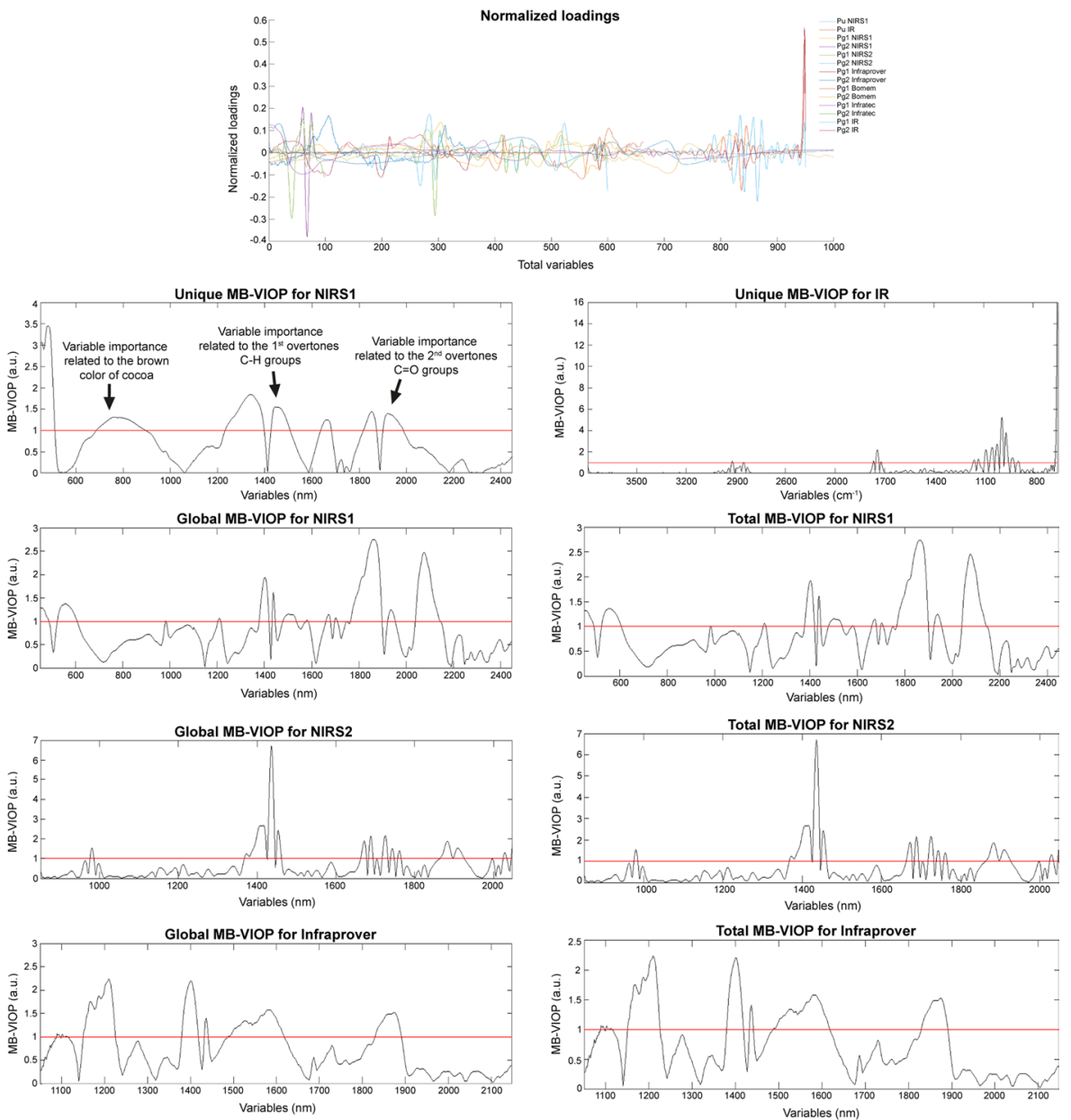

Global MB-VIOP for Bomem
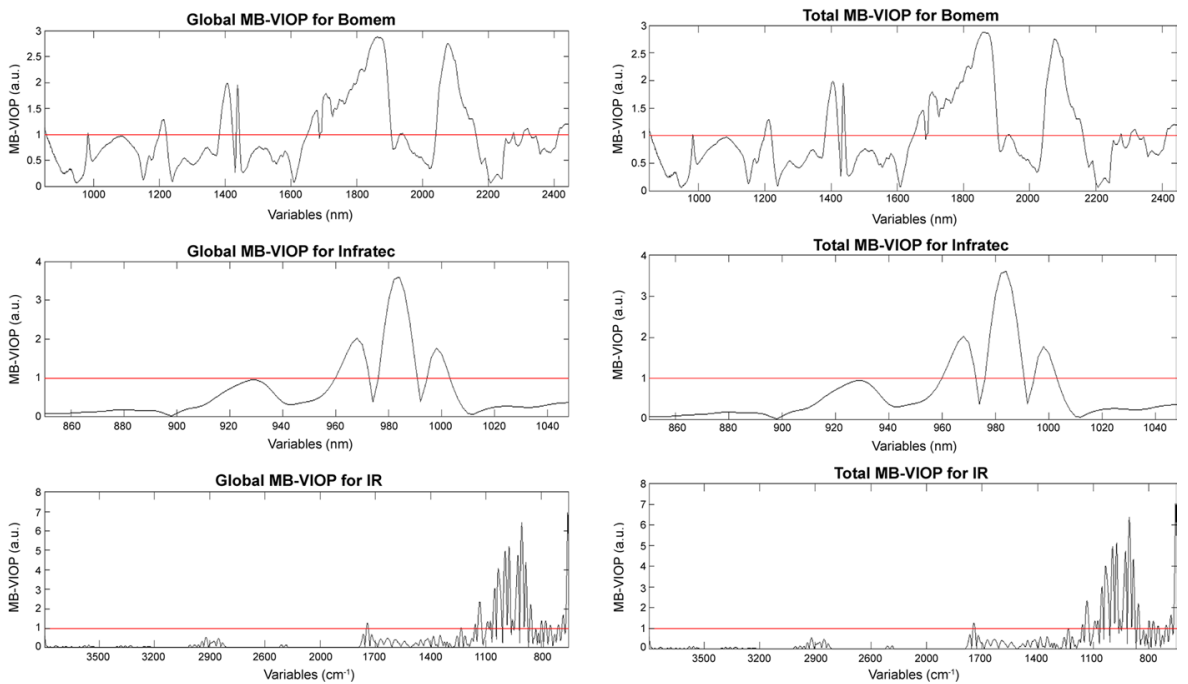

Fig. $3 \mathrm{MB}-\mathrm{VIOP}$ results for the marzipan dataset. The normalized loadings (for all the blocks and components) obtained from the OnPLS model are provided on the top. The unique, global and total MB-VIOP plots are also provided, including the threshold line at $\mathrm{MB}-\mathrm{VIOP}=1$. The variables determined as relevant by the $\mathrm{MB}-\mathrm{VIOP}$ algorithm have been annotated in the unique MB-VIOP plot for the data block NIRS1 according to the organic compound of marzipan and/or cocoa that they help to explain 
variables were very similar to the MB-VIOP results for the total variation, as can be seen by comparison of the plots in Fig. 3.

Giving an overall look at the MB-VIOP plots of Fig. 3, the manifest variables selected as relevant for the two global latent variables (global model components) seemed to relate to (i) the sugar content (majorly sucrose, but also small amounts of invert sugar and glucose syrup), and (ii) the almonds and apricot kernels. The unique MB-VIOP plots were related to special and unique characteristics of some marzipan samples and/or some spectrometers, as it will be explained in this section.

Block NIRS1 contains measurements done using an instrument that was able to cover, not only the NIR region, but also the visual light range (400-800 nm). Thanks to this, differences in color could be detected for the marzipan samples. Interestingly, MB-VIOP determined that some variables corresponding to the range between 450 and $800 \mathrm{~nm}$ (visual light region) were relevant for explaining variation only detectable in NIRS1 (i.e., unique for this data block). These important variables relate to the cocoa that was added to some marzipan samples (they had a more brownish color). Besides, by looking at the whole unique MB-VIOP plot (from 450 to $2448 \mathrm{~nm}$ ) in Fig. 3, it can be seen that, aside from the variables with high MB-VIOP values detected in the visual light range, there were also important variables located at 1232-1396 nm, 1428-1506 nm, 1638-1682 nm, 1818-1872 nm, and 1902-1986 nm. The cocoa NIR spectrum has been described in the literature [52], thus by matching of some of the important wavelengths found by MBVIOP and the known composition of the cocoa, it is possible to realize the enhanced and easier model interpretation achieved by using MB-VIOP (which is not possible by using the OnPLS model loadings provided in Fig. 3). The wavelengths at $1478-1506 \mathrm{~nm}$ are important to uncover the OnPLS model variation related to the first overtones of the $\mathrm{C}-\mathrm{H}$ groups of the cocoa, and variables at 1902-1986 nm explain the variation related to the second overtones of the $\mathrm{C}=\mathrm{O}$ groups of the cocoa (see Fig. 3).

The Infratec MB-VIOP revealed three clear regions of important variables located at 960-972 nm, 978-990 nm and 996-1002 nm (see MB-VIOP plots for Infratec in Fig. 3). These variables are selected as relevant by the MB-VIOP algorithm because they are related to the carbohydrates, proteins, water and lipids (i.e., the second overtones of $\mathrm{O}-\mathrm{H}$ and $\mathrm{N}-\mathrm{H}$ stretching vibrations, and the third overtones of $\mathrm{C}-\mathrm{H}$ stretching vibrations). These substances are common to all the marzipan samples, which explains that these wavelengths (variables) were highlighted in the global MB-VIOP plot. It is worth noticing that these three wavelength regions can be also seen (albeit not so clearly) in the MB-VIOP plots of NIRS2.

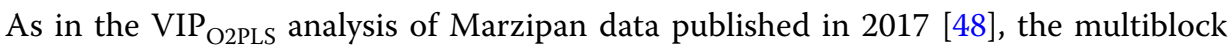
model generated for the VIP analysis is only between spectra, not between spectra and concentrations; which can be unusual, but also useful either for technical reasons (e.g., to compare spectrometers) or for spectroscopic reasons (e.g., to see the correspondence between bands in IR and bands in NIR - overtones -). The MB-VIOP plots for NIRS1 and Bomem (Fig. 3) were very similar because of the characteristics that the NIR spectrometers had in common, however MB-VIOP found some differences in the variable importance that could (maybe) be attributable to the different optical principles of the two instruments (dispersive scanning for the NIRS1, and FT interferometer for the Bomem). On the other hand, the IR data block contained relevant 
variables (wavenumbers) that explained information that is unique for this block, due to the differences in type of spectroscopy (IR/NIR) and instrumentation (spectrometer components).

Some very intense peaks in the MB-VIOP plots correspond to variables that are important for some major marzipan compounds. For example, the peak around $1440 \mathrm{~nm}$ in the MB-VIOP plot for NIRS2 could be related to the O-H bonds, and the peak around $2100 \mathrm{~nm}$ in the MB-VIOP plot for Bomem could relate to the protein amino acids.

\section{Selection of the most relevant variables in systems biology multiblock analysis for enhanced model interpretation and dimensionality reduction}

For the Hybrid Aspen data, the variables were sorted by importance using MB-VIOP, and afterwards, this information was used for achievement of enhanced interpretability (higher percentage of explained model variation) and reduced model dimensions (less variables). The purpose was not only to validate MB-VIOP as a method for variable importance sorting, but also for multiblock variable selection. To this end, two MB-VIOP variable selections (both of them from the original model, i.e. not sequentially done) were carried out, one choosing the variables with MB-VIOP values over the default threshold (MB-VIOP $\geq 1$ ), and another variable selection with a more conservative criterion (i.e., $M B-V I O P \geq 0.5$ ). Afterwards, two new OnPLS models were generated using only the variables selected by MB-VIOP; the number of variables used in the original and the two new reduced multiblock models, as well as the percentages of total explained variation, are summarized in Table 4. We want to emphasize that the MB-VIOP profile used for selecting the variables was the total MB-VIOP because the goal was to improve the total model interpretation without focusing on any concrete part of the model. Nevertheless, it would be possible to select the variables that are more convenient for improving the interpretation of a specific type of variation (e.g., the local variation) by using its corresponding MB-VIOP profile (e.g., the local MB-VIOP) and building a new model with this selected subset of variables; hereby, MB-VIOP is a variable selection method à la carte according to the part of the model (total, global, local or

Table 4 Summary of the number of variables used for the OnPLS models (the original and the two reduced models) and the percentages of explained total variation for the Hybrid Aspen data

\begin{tabular}{llll}
\hline Data & OnPLS models & Number of variables used & $\begin{array}{l}\text { Explained total } \\
\text { variation (\%) }\end{array}$ \\
\hline Transcript & Original & 14,738 & 75.0 \\
& Total $M B-V I O P \geq 0.5$ & 13,127 & 80.1 \\
& Total $M B-V I O P \geq 1.0$ & 4452 & 85.2 \\
Protein & Original & 3132 & 55.0 \\
& Total MB-VIOP $\geq 0.5$ & 2186 & 67.3 \\
Metabolite & Total MB-VIOP $\geq 1.0$ & 683 & 71.6 \\
& Original & 281 & 58.3 \\
& Total $M B-V I O P \geq 0.5$ & 232 & 65.5 \\
& Total $M B-V I O P \geq 1.0$ & 81 & 76.2 \\
\hline
\end{tabular}

The information has been distributed in three areas according to data block (transcriptomics, proteomics and metabolomics), and each area is divided in three rows: one for the original model, one for the reduced model using the variables with total $\mathrm{MB}-\mathrm{VIOP} \geq 0.5$, and one for the reduced model using the variables with total $\mathrm{MB}-\mathrm{VIOP} \geq 1$ 
unique) targeted to be improved. In order to show possible sensitivity differences among MB-VIOP profiles due to threshold choice (i.e., MB-VIOP $\geq 1$ or $\mathrm{MB}-\mathrm{VIOP} \geq 0.5$ ), the number of selected variables is shown in Additional file 1: Table S1 in the Supporting Information and as bar plots in Fig. 4 for each type of variation and each threshold choice. From Fig. 4, it does not seem to exist significant differences between total and global profiles in relation to the number of selected variables. However, the number of variables selected when using the threshold $\mathrm{MB}-\mathrm{VIOP} \geq 1$ (blue bars in Fig. 4) was clearly lower than when using the threshold $\mathrm{MB}-\mathrm{VIOP} \geq 0.5$ (green bars in Fig. 4). For the unique variance, the reduction of number of selected variables using $\mathrm{MB}-\mathrm{VIOP} \geq 0.5$ was substantially more significant than for the joint variation types.

The blocks of the original OnPLS model contained 14,738 microarray elements (variables of the transcriptomics data block) that explained the $75.0 \%$ of total variation, 3132 extracted chromatographic peaks (variables of the proteomics data block) that explained the $55.0 \%$ of total variation, and 281 extracted chromatographic peaks (variables of the metabolomics data block) that explained the $58.3 \%$ of total variation. After performing a conservative (i.e., with threshold at 0.5 a.u.) MB-VIOP selection of variables, a subset of variables was used for building a new multiblock model obtaining an increase of model interpretability; as shown in Table 4, 13,127 variables from the transcriptomics data explained the $80.1 \%$ of total variation, 2186 variables from the proteomics data explained the $67.3 \%$, and 232 variables from the metabolomics data explained the $65.5 \%$. The second new multiblock model with reduced dimensions (using MB-VIOP $\geq 1$ as criterion for selecting the subset of variables) had substantially less variables (approximately, 1/3 of the original ones) and, at the same time, increased the interpretability (measured as percentage of explained total variation in Table 4); more specifically, only 4452 transcript variables were needed to explain the $85.2 \%$ of total variation, 683 protein variables explained the $71.6 \%$, and 81 metabolite variables the $76.2 \%$. Due to the latter improvement, a deep exploration of the forty most important variables of each block, for interpreting the total multiblock model, was carried out. The identification of these variables is provided in Additional file 1: Table S2 for each block.

The variables with global MB-VIOP values above the threshold (Additional file 1: Table S3) are important for explaining the variation related to common characteristics of the growth processes of the plants, as well as both the genotype and the internode effects (common to all data blocks). Some of the most important variables to explain this

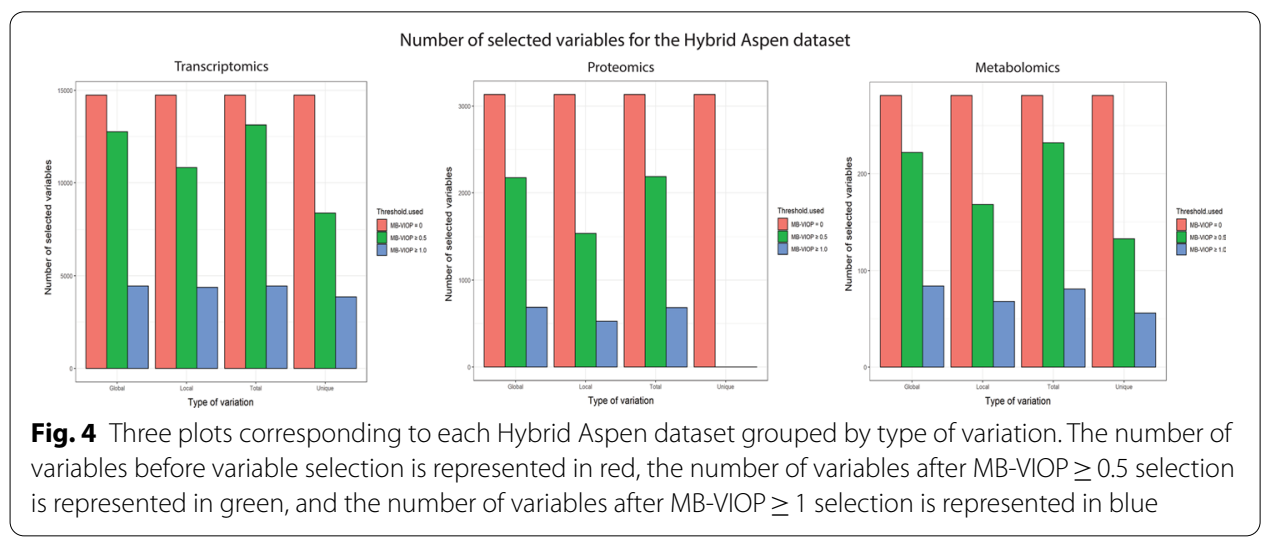


latent information were PU07944 from the transcript data, the protein variables 966 and 1071, and Win022_C04 from the metabolite data.

MB-VIOP determined that the PU06931 was the most important microarray element for explaining the locally joint information, related to lignin biosynthesis, between the transcript and the protein data, with a local MB-VIOP value of 8.05 a.u. (Additional file 1: Table S4), followed by PU07326 and PU06434; whilst for explaining the locally shared information with the metabolite data, the most important microarray elements were PU00630 (4.50 a.u.), PU03044 and PU22639. Connecting to, variable 966 (local MB-VIOP value equal to 9.76 a.u.), followed by variables 2121 and 1115, were the most important protein variables for explaining the variation locally shared with the transcriptomics block. In the metabolite space, variable Win031_C01 (5.39 a.u.), followed by Win021_C05 and Win034_C06, were selected as the most relevant metabolite variables for explaining the local variation shared with the transcript data.

The housekeeping-like events, and the differences between the instrumentation used to characterize the data in the three different platforms, were uncovered by the variables listed in Additional file 1: Table S5 (i.e., the variables with higher values of unique MB-VIOP).

In order to explore the possibility of finding variables that could explain more than one type of variation (i.e., the special cases illustrated in Fig. 1), it is worth comparing the tables and plots for the unique, local and global MB-VIOP values. For example, in this biological case, the variable Win021_C05 of the metabolomics data block helps to explain variation that is globally shared by all the data blocks, and also contributes to explain variation that is locally shared only between the metabolomics and the transcriptomics data blocks. Therefore, one variable can contain information related to more than one type of variation, and MB-VIOP is able to detect and distinguish this feature.

\section{Comparison of MB-VIOP to MOFA and block-sPLS}

Two unsupervised variable selection methods, i.e. block sparse partial least squares (block-sPLS) and multi-omics factor analysis (MOFA), have been compared to multiblock variable influence on orthogonal projections (MB-VIOP). All three methods have been run in symmetric mode, i.e. giving the same importance to all data blocks and considering all of them as descriptor matrices. The results have been evaluated and we present the highlighted remarks of the comparison in this section. Further details about the procedures and calculations are described in section "Determination of variable importance in block-sPLS and MOFA for comparison to MB-VIOP variable selection".

\section{MB-VIOP and MOFA comparison for synthetic data and real omics data}

In order to compare the performance of MB-VIOP and MOFA, an 8-component MOFA model was generated yielding a percentage of total explained variation of $54.5 \%$ for D1, 100\% for D2, 100\% for D3 and 80\% for D4; i.e., similar to the percentage of total explained variation obtained by MB-VIOP $\left(85.8 \%\right.$ for $\mathbf{D}_{1}, 100 \%$ for $\mathbf{D}_{2}, 100 \%$ for $\mathbf{D}_{\mathbf{3}}$ and $80 \%$ for $\mathbf{D}_{4}$ ). The distribution of the model components had similarities and differences in relation to the one obtained by MB-VIOP. Whilst MB-VIOP found two global components and three local components as expected from the design of the synthetic data, MOFA found 3 global components and three local components (see Additional file 1: 
Figure S1). For the local variation, both methods found the local components shared by D2-D3-D4 and D1-D2, but yielded different local assessments for the other latent variables. There were also differences in the discovering of the unique components; however, both methods found a unique component for D1. In general, it seems that MB-VIOP assessed better the explained variation per model component than MOFA.

Interestingly, the results of the variable selection performed by MOFA shared many similarities with MB-VIOP. When looking at the absolute MOFA loadings for the first global component, most of the variables selected by MOFA for the four data blocks were the same variables selected by MB-VIOP (marked in purple in Fig. 2). The second and third components of MOFA contained a mix in the selection of the variables that seemed to partially match the variables selected by MB-VIOP for the second global component (marked in grey in Fig. 2). There was also similarity in the selected variables from both methods when looking at the explained local variation, e.g. the same variables were selected as important in the absolute loadings assessment for the fourth component of MOFA and the local D1-D2 component of MB-VIOP (marked in orange in Fig. 2). The evaluation of the variable selection for the unique components found by both methods, i.e. for the unique components of D1 (in pink in Fig. 2), also showed a similar variable importance assessment; however, MOFA did not highlight variable 13 that helps to explain two unique components (as explained in section "Evidence of the reliability and the efficiency of MB-VIOP using synthetic data") over the variables that were only helping to interpret one unique component. As an example of how the assessment has been visualized in MOFA, the absolute loading plot from MOFA for the latter example has been included as Additional file 1: Figure S2.

For the Hybrid Aspen case, MOFA yielded 8 model components (see Additional file 1: Figure S3). The total variation explained by the model was $24.6 \%$ for metabolomics, $29.5 \%$ for proteomics and $69.2 \%$ for transcriptomics. The MOFA algorithm found two global components and two unique components for the transcriptomics and the proteomics data. It also uncovered local variation shared by the transcriptomics and the metabolomics data. However, the components distribution seems difficult to assess by looking at Additional file 1: Figure S3 due to the low values of the R2 parameter for some cases.

The variable importance assessment performed using MOFA shared some similarities with the one performed using MB-VIOP. For instance, the metabolites ranked as the most important ones in the MOFA model (e.g. Win022_C04, Win020_C03, Win009_C09, Win034_C06, Win031_C01 or Win021_C05) were selected as important top variables to explain global variation in both MB-VIOP (Additional file 1: Table S3 and section "Selection of the most relevant variables in systems biology multiblock analysis for enhanced model interpretation and dimensionality reduction") and MOFA (Additional file 1: Figures S4-S5). The variable selection for the transcripts and the proteins was also consistent for both MB-VIOP and MOFA; e.g. top selected transcripts for explaining the unique variation in MB-VIOP (such as PU27903 or PU28218) were also determined as important by MOFA, and proteins such as 847 or 270 were also selected in both methods. For the total models, the same 2239 transcripts, 175 proteins and 32 metabolites were selected as important features by both methods. 


\section{MB-VIOP and block-sPLS comparison for the Hybrid Aspen data}

For the comparison between the MB-VIOP and the block-sPLS methods, the number of variables used in the original and reduced models and the total explained variation are summarized in Tables 4-5. Both methods, as specified in section "Determination of variable importance in block-sPLS and MOFA for comparison to MB-VIOP variable selection", used similar specifications (such as the number of components for explaining the predictive variation or the constraint/penalization degree). The percentages of explained variation obtained by the block-sPLS algorithm were inferior to the ones obtained by MB-VIOP. MB-VIOP was able to explain more total variance than block-sPLS. Furthermore, when generating the models with a reduced number of variables, MB-VIOP improved the percentage of explained variation by using only the subset of MB-VIOP selected variables for the new models instead of all original variables. On the contrary, the reduced models generated by block-sPLS explained less variance than the original block-sPLS model.

The overlap between the selected variables by MB-VIOP and block-sPLS was assessed. For the moderately constrained (threshold of 0.5 a.u.) reduced MB-VIOP and blocksPLS models, the same 4257 transcripts, 559 proteins, and 75 metabolites, were selected by both methods as important. For the normally constrained (threshold of 1.0 a.u.) reduced MB-VIOP and block-sPLS models, the same 2053 transcripts, 207 proteins, and 33 metabolites, were selected by both methods as important. Considering the total number of variables selected by both methods (see Tables 4-5), this seems a good overlap for the variable selection performed using MB-VIOP and block-sPLS. Besides, some variables mentioned in section Selection of the most relevant variables in systems biology multiblock analysis for enhanced model interpretation and dimensionality reduction were selected by both methods as important for interpreting the joint variation. For example, both MB-VIOP and block-sPLS selected Win022_C04 as the most important variable in the metabolomics data, and proteins such as 1071, or transcripts such as PU07944, we selected for the proteomics and the transcriptomics data respectively.

Table 5 Summary of the number of variables used for the block-SPLS models (the original and the two reduced models) and the percentages of explained total variation for the Hybrid Aspen data

\begin{tabular}{llll}
\hline Data & Block-sPLS models & $\begin{array}{l}\text { Number of } \\
\text { variables used }\end{array}$ & $\begin{array}{l}\text { Explained total } \\
\text { variation (\%) }\end{array}$ \\
\hline Transcript & Original block-sPLS & 14,738 & 68.0 \\
& Block-sPLS comparable to MB-VIOPtot $\geq 0.5$ model & 13,151 & 68.0 \\
& Block-sPLS comparable to MB-VIOPtot $\geq 1.0$ model & 4483 & 66.0 \\
Protein & Original block-sPLS & 3132 & 50.0 \\
& Block-sPLS comparable to MB-VIOPtot $\geq 0.5$ model & 2201 & 50.0 \\
& Block-sPLS comparable to MB-VIOPtot $\geq 1.0$ model & 685 & 48.0 \\
Metabolite & Original block-sPLS & 281 & 54.0 \\
& Block-sPLS comparable to MB-VIOPtot $\geq 0.5$ model & 236 & 54.0 \\
& Block-sPLS comparable to MB-VIOPtot $\geq 1.0$ model & 77 & 52.0 \\
\hline
\end{tabular}

The information has been distributed in three areas according to data block (transcriptomics, proteomics and metabolomics), and each area is divided in three rows: one for the original model, one for the reduced model using a constraint degree similar to the total $\mathrm{MB}-\mathrm{VIOP} \geq 0.5$, and one for the reduced model using a constraint degree similar to the total $\mathrm{MB}-\mathrm{VIOP} \geq 1$ 


\section{Conclusions}

A novel multiblock variable selection method, called multiblock variable influence on orthogonal projections ( $M B-V I O P$ ), has been tested and validated here. Evidence of its reliability, efficiency and usefulness have been shown. MB-VIOP can assess in a reliable and efficient way the importance of both isolated and ranges of variables in any type of data. Furthermore, MB-VIOP can deal with strong overlapping of types of variation, as well as with many data blocks with very different dimensionality. In addition, MB-VIOP connects the variables of different data matrices according to their relevance for the data interpretation of each latent variable (component) of an OnPLS model.

MB-VIOP also takes advantage of the full symmetry of the OnPLS model, which points at some advantages over the combination of sequential multiblock modelling techniques and variable selection methods. In sequential multiblock regression, even if the parameters keep the information of all parts of the sequence (i.e., other blocks of the multiblock dataset), the sequential approach only allows the weighting of the variables in a unique path (sequence) previously established, without any symmetry. Thus, the possibility of taking into account shared influences of the variables in other combinations, not considered by the pre-established path, is missing. MB-VIOP uses the symmetry of OnPLS for establishing fairer relationships/influences between variables of different blocks iterating over all components and all blocks, i.e. considering all combinations. In addition, it is worth emphasizing the ability of VIP ${ }_{\text {OPLS }}$ [39], VIP O2PLS $_{\text {[48] }}$ and MB-VIOP to uncover the variables that are important for the uncorrelated (orthogonal) variation. However, for enhanced model interpretability, the synthetic example (section Evidence of the reliability and the efficiency of MB-VIOP using synthetic data) has shown how MB-VIOP surpasses any try of variable importance assessment done by means of OnPLS p loadings. More specifically, MB-VIOP provides a correctly proportionated importance assessment of the variables, even when the profiles are affected by high overlapping or when there is an outsizing number of variables related to a specific type of variation, assessment that cannot be achieved by the normalized OnPLS loadings.

MB-VIOP has been compared to block-sPLS and MOFA multiblock methods. Even if the comparisons are limited by the component distribution assessed by each method, the modelling and variable selection performed led to interesting conclusions. In relation to the modelling, MB-VIOP explained a higher percentage of total variation than MOFA and block-sPLS. For the feature selection, when using synthetic data, the variables selected by MB-VIOP and MOFA seemed to be consistent; however, when using real omics data, even if some of the most important variables were selected in both methods, differences in the final sorting seemed to rise when the values of the weights of the ranked variables were too adjusted. The overlapping of selected variables between block-sPLS and MB-VIOP, and MOFA and MB-VIOP, were both significant, consistent, and similar in number of variables. It is also worth mentioning, that MB-VIOP was able to keep the proportionality in the variable importance assessment (e.g., showed as a peak variable 13 of the synthetic data because of explaining more variation than the other variables); however, MOFA did not keep this proportionality as explained in the Results section.

Nevertheless, it is interesting to compare the results for the Marzipan example obtained here with the ones obtained in 2017 [48], for the NIRS2 and the IR data blocks, 
using an O2PLS model and the VIP $\mathrm{O}_{\mathrm{O} P L S}$ variable selection method. As expected, the importance assessments are very similar. However, the absence of the other four data

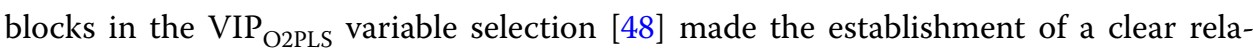
tionship between the variables of the two present blocks and the variables of the four absent blocks totally impossible, which led to classify those variables as containers of orthogonal variation; however, when the variable assessment was performed in a sixblock multiblock analysis with MB-VIOP, the same variables were selected as relevant for explaining variation shared between NIRS2 and the other data blocks (e.g., variables around $1200 \mathrm{~nm}, 1400 \mathrm{~nm}$ and $1800 \mathrm{~nm}$ ). Hereby, when using all the blocks in a full multiblock system, the assessment was improved in relation to the two-block combination analysis.

MB-VIOP was able to reduce the number of variables of an OnPLS model (in a third for the Hybrid Aspen example) and, at the same time, increase the model interpretability. Besides, it has been shown that MB-VIOP is a variable selection method $\grave{a}$ la carte for OnPLS models that allows to target a concrete type of variation (global, local or unique), or, if desired, target the total model, for afterwards building a stronger reduced OnPLS model with better interpretability than the original model.

The above achievements entail valuable advantages for industry and research groups (e.g., time optimization, fast and reliable variable selection, or enhanced interpretation in multiblock analysis). We envisage the use of MB-VIOP in fields like chemistry, biology, medicine, psychology, economy, physics, cybernetics, and engineering, inter alia. Since VIP ${ }_{\text {OPLS }}$ [39] can be applied to both OPLS ${ }^{\circledR}$ and PLS models, it is expected by the authors that MB-VIOP could be successfully applied not only to OnPLS models but also to multiblock PLS (e.g., MBPLS and HPLS models). This should lead to a more reliable and accurate variable sorting/selection in the MBPLS analysis than using other methods because of the more efficient and detailed weighting of the variables (especially due to the further connectivity ability, and the use of not only the amount of variation in $\mathrm{Y}$ explained by the model -SSY- but also the explained amount of variation in X -SSX-) of MB-VIOP compared to PLS-VIP (VIP ${ }_{\text {PLS }}$ ) method applied to multiblock analysis. The verification of the latter hypotheses is part of future work.

\section{Methods}

\section{General notation}

Scalars are written using italic characters (e.g. $h$, and $H$ ), vectors are typed in bold lowercase characters (e.g. h), and matrices are defined as bold upper-case characters (e.g. H). When necessary, the dimensions of the matrices are specified by the subscript $r x$ $c$, where $r$ is the number of rows and $c$ is the number of columns. Transposed matrices are marked with the superscript $T$. The symbol $\circ$ indicates a Hadamard power or product. Matrix elements are represented by the corresponding matrix italic lower-case character adding as subscripts the row and the column where they are located (e.g., for an $\mathbf{H}$ matrix, an element located in row $i$ and column $k$ would be indicated as $h_{i k}$ ). Model components are represented by $a$. Subscripts $g, l$ and $u$ stand for global, local and unique respectively. The units $a . u$. stand for arbitrary units for the MB-VIOP values. Notation referring to specific cases is explained insitu. 


\section{Determination of the variable importance in OnPLS models}

MB-VIOP is a model based variable selection method that uses a number $n$ of preprocessed data matrices $(\mathbf{D})$, and the scores $(\mathbf{t})$ and the normalized loadings $(\mathbf{p})$ from an OnPLS model. The Hadamard products of the normalized loadings (denoted as $\mathbf{p}^{\mathrm{O}}$, i.e. $\mathbf{p} \circ \mathbf{p}$ ) are computed, and afterwards, they are multiplied by the ratio between the variation explained by the corresponding model component and the cumulated variation. The latter sum of squares (SS) ratio helps to assess the variable importance focusing on interpretability, i.e. the SS ratio helps to know which variables are more helpful to explain the maximum amount of variation. The scores are used for the calculation of the residuals prior to computation of the sum of squares. The MB-VIOP values, which will conform the MB-VIOP vectors, are obtained by iterative calculations among both the components (latent variables) and the data matrices, with specific combinations according to the type of variation. As final step, the square root is taken, and a normalization is performed by applying the Euclidean norm (2-norm) and multiplying by the number of manifest variables raised to the $1 / 2$ power. The latter explanation is the general procedure for all types of variation (see Fig. 1), details and specifications are provided below. We also describe the calculations, equations (for the unique, the local, the global, and the total variations), and how to interpret the results provided by the MB-VIOP algorithm, in the subsequent sections.

\section{Threshold of MB-VIOP values for importance assessment}

The threshold for importance assessment according to the MB-VIOP values is simi-

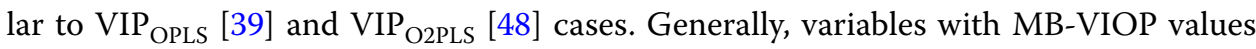
higher than 1 are considered important for the model interpretation, whereas variables with MB-VIOP values below 1 could be considered irrelevant. Since the sum of squares of all MB-VIOP values is equal to the number of manifest variables of the respective data matrix, the average MB-VIOP is equal to 1 ; therefore, if all variables would have the same contribution to the OnPLS model, they would have MB-VIOP values equal to 1. The threshold is represented in all plots by a red horizontal line at MB-VIOP $=1$ for fast visual assessment. However, since this is a data-driven methodology, there can be special cases that justify the use of other threshold values according to either the goal of the variable selection or the demand level of dimensionality reduction, as shown in section "Selection of the most relevant variables in systems biology multiblock analysis for enhanced model interpretation and dimensionality reduction.

\section{Calculation of MB-VIOP for the unique components}

The first computation performed in the algorithm is the unique MB-VIOP (Eq. 1), which allows to assess the importance of the variables related to the unique information contained in each data block. It is worth noting that the unique information contained in the unique variation (exclusive of one block, i.e. not shared with other blocks) can be elucidated focusing on a reduced subset of important variables selected by MB-VIOP without need to inspect all variables. This subset of important variables is found using Eq. 1. 


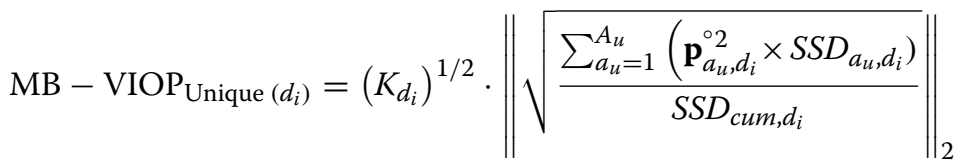

In Eq. $1, d_{i}$ indicates which data block we are referring to, $K$ is the number of manifest (input) variables of the data block, $A_{u}$ represents the total number of unique components (unique latent variables), $a_{u}$ indicates a specific unique component, $\mathbf{p}$ corresponds to the normalized loadings extracted from the OnPLS model, $S S D_{a u, d i}$ stands for sum of squares of a data block for an $a_{u}{ }^{\text {th }}$ component, $S S D_{\text {cum }, d i}$ stands for the cumulated sum of squares of a data block, and the Euclidean normalization is indicated using the subscript 2 and enclosing the normalized expression between double-line brackets.

\section{Calculation of MB-VIOP for the local components}

MB-VIOP ${ }_{\text {Local }}$ gives values higher than 1 to those input variables that are important for explaining the variation (information) of a specific local component in an OnPLS model. The local MB-VIOP (Eq. 2) is calculated iterating among all the local components, selecting the blocks that have variables locally connected (see Fig. 1), and leaving out any data block that is related to either global variation or local variation linked to a different local component. Furthermore, the local part of the MB-VIOP algorithm is constrained to ignore the connection of a data block with itself, since this would increase the importance of the locally connected variables in relation to the whole model variable influence, making the weighting system unfairly favorable to the variables with locally shared information.

In Eq. 2, the local MB-VIOP calculation is summarized. The calculation iterates among all the local components $A_{l}$, and the local MB-VIOP values for each local component are calculated considering all the combinations (direct and reverse) of the locally connected blocks, here denoted $D_{L C}$. It should be mentioned that $D_{L C}$ includes the data block $d_{i}$ and also the blocks connected to it $\left(d_{L C}\right)$ in Eq. 2. For instance, in a multiblock analysis involving four or more data blocks, if the variation of a local component is shared by three blocks, the corresponding local MB-VIOP values will be calculated using exclusively these three blocks in an iterative and exchangeable way either to provide the normalized loading (p) or to provide the sum of squares values (SSD). In the end, all three connected blocks will have contributed as both $d_{i}$ and $d_{L C}$ according to the specific ongoing calculation.

$$
\mathrm{MB}-\operatorname{VIOP}_{\text {Local }\left(d_{i}\right)}=\left(K_{d_{i}}\right)^{1 / 2} \cdot\left\|\sqrt{\beta^{-1} \cdot\left(\frac{\sum_{a_{l}=1}^{A_{l}} \sum_{d_{L C}=1}^{D_{L C}}\left(\mathbf{p}_{a_{l}, d_{i}}^{2} \times S S D_{a_{l}, d_{L C}}\right)}{S S D_{\text {cum }, d_{L C}}}\right)}\right\|_{2}
$$

The iterative computation of the local MB-VIOP is condensed in Eq. 2, where $A_{l}$ represents the total number of local components, $a_{l}$ stands for a specific local component, $\beta$ (beta) represents the connectivity degree, $S S D_{a l, d L C}$ stands for sum of squares explained by an $a_{l}^{\text {th }}$ component for a data block $d_{L C}, S S D_{\text {cum }, d L C}$ is the cumulated sum of squares of 
the data block $d_{L C}$. The rest of nomenclature is analogous to section "Calculation of MBVIOP for the unique components".

The connectivity degree $\beta$ is based on the number of local connections, which makes MB-VIOP different from VIP $\mathrm{O}_{\mathrm{O} P L S}$, since the latter uses the number of local components. It is worth noting that in $\mathrm{VIP}_{\mathrm{O} 2 \mathrm{PLS}}$ the number of local components will always be equal to the number of local connections among blocks since there are only two-block connections (since O2PLS cannot handle more than two blocks). However, in MB-VIOP, there can be connections among more than two blocks related to the same local component, which implies that the number of local components will not match the number of connections. Hereby, the connectivity degree is different in MB-VIOP.

\section{Calculation of MB-VIOP for the global components}

$\mathrm{MB}-\mathrm{VIOP}_{\mathrm{Global}}$ pinpoints the variables that are relevant for explaining the variation (information) that is shared by all the data blocks related to a specific global component (these variables would be the ones filled in white inside the grey zone of Fig. 1), e.g., a common biological effect present in all data matrices. The global MB-VIOP (Eq. 3) is calculated by iterating over all the data block combinations (direct and reverse modes) and all the global components. In Eq. 3, for a more intuitive explanation, $d_{i}$ is used as the data block to which the normalized loading of an iteration belongs, and $d_{j}$ as the data block to which the SSD values of an iteration belong. The blocks exchange these roles on the spot (i.e., at the exact iteration corresponding to a specific calculation); thus, all D data blocks are used as both $d_{i}$ and $d_{j}$, but in different moments of the global MB-VIOP computation.

$$
\mathrm{MB}-\operatorname{VIOP}_{\text {Global }\left(d_{i}\right)}=\left(K_{d_{i}}\right)^{1 / 2} \cdot\left\|\sqrt{\frac{\sum_{a_{g}=1}^{A_{g}} \sum_{d_{j}=1}^{D_{j}=D}\left(\mathbf{p}_{a_{g}, d_{i}}^{\circ 2} \times S S D_{a_{g}, d_{j}}\right)}{S S D_{\text {cum }, d_{j}}}}\right\|_{2}
$$

In Eq. 3, $A_{g}$ represents the total number of global components (global latent variables), $a_{g}$ indicates a specific global component, $S S D_{a g, d j}$ stands for sum of squares of an $a_{g}^{\text {th }}$ component related to a data block $d_{j}$, and $S S D_{\text {cum, } d j}$ stands for the cumulated sum of squares of the data block $d_{j}$, and the rest of nomenclature is analogous to Eqs. 1 and 2.

\section{Calculation for the total variable influence for interpreting the whole model}

The overview of which variables are more relevant for the total model interpretation (i.e., considering the global, the local and the unique variations involved in the OnPLS model) is highly appreciated in industrial environments; this is achieved by MB-VIOP ${ }_{\text {Total }}$ In the total MB-VIOP the contributions of the global, local and unique MB-VIOP vectors are joined achieving a proper weighting of all variables for the total variable influence on all projections. Equation 4 summarizes its computation.

$$
\mathrm{MB}-\operatorname{VIOP}_{\text {Total }\left(d_{i}\right)}=\left(K_{d_{i}}\right)^{1 / 2} \cdot\left\|\sqrt{\left(\mathrm{MB}-\operatorname{VIOP}_{\text {Unique }\left(d_{i}\right)}\right)^{2}+\left(\mathrm{MB}-\operatorname{VIOP}_{\text {Local }\left(d_{i}\right)}\right)^{2}+\left(\mathrm{MB}-\operatorname{VIOP}_{\mathrm{Global}}\left(d_{i}\right)\right)^{2}}\right\|_{2}
$$


The nomenclature of Eq. 4 is analogous to the nomenclature mentioned in the previous sections. As in the other cases, MB-VIOP leads to a vector which contains the MBVIOP values for the variables of each data block (but the calculations take all blocks into consideration). As it will be explained in section "Graphical representation of the MBVIOP results for variable importance assessment", the visualization by plotting the MBVIOP vectors for each block is one of the various options.

\section{Graphical representation of the $M B-V I O P$ results for variable importance assessment}

Equations 1-4 lead to four MB-VIOP vectors (i.e., MB-VIOP ${ }_{\text {Unique, }}, \mathrm{MB}^{-V_{I O P}} \mathrm{Loca}_{\text {, }}$ MB$\left.\mathrm{VIOP}_{\text {Global }}, \mathrm{MB}-\mathrm{VIOP}_{\text {Total }}\right)$. It is always possible to look at the numerical values of MBVIOP for each variable of the OnPLS model to assess their importance for the data interpretation. However, this can become a very time-consuming and painstaking task. Hence, a reduced table containing only target variables and its MB-VIOP values, or a graphical representation of these $\mathrm{MB}-\mathrm{VIOP}$ vectors, seem a more convenient way to present the results. The MATLAB code created for MB-VIOP allows several ways to plot the results; for this paper, block-wise plots have been chosen (even though the calculation of each MB-VIOP has involved all the data blocks because of being a multiblock variable sorting). Other graphical representations could be possible; in a case where all data blocks of the OnPLS model would contain the same manifest variables, it would be possible to make a $3 \mathrm{D}$ (cube) plot locating the manifest variables on the $\mathrm{X}$-axis, labeling the data blocks on the Y-axis, and inserting the MB-VIOP values on the Z-axis (the vertical one); this visualization becomes ideal for matrices with the same variables (e.g., in some comparison studies), but it is not recommended when the data blocks have different variables (which is frequently the case).

In section "Results and discussion", the results were represented visualizing the MBVIOP values for each data block (by rows in the figures), and for type of variation interpreted by the variables (by columns); thus, each column of plots separately represents the unique, the local, the global and the total MB-VIOP results (Fig. 2 can be used as an example). As mentioned in section "Threshold of MB-VIOP values for importance assessment", a threshold at MB-VIOP $=1$ (represented by a red horizontal line) is included in each plot; variables with values above the red line are relevant for the interpretation of the type of variation corresponding to the plotted MB-VIOP. The variables of different blocks that contribute to explain the same variation (e.g., a common biological effect among data blocks, or a common feature of several instruments) are marked with the same color in all block-wise plots (see Fig. 2).

\section{Determination of variable importance in block-SPLS and MOFA for comparison to MB-VIOP variable selection.}

Variable importance assessment using MOFA on the SD16-365GLU and the Hybrid Aspen data MOFA [33] performs unsupervised data integration aiming to uncover the principal sources of variation in multi-omics datasets, and, in some aspects, it can be seen as a statistical generalization of principal component analysis for omics data. MOFA infers a set of factors (model components) that contain biological or technical variation that can be either shared by multiple data matrices or unique of a specific data matrix. MOFA 
achieves factor-wise sparsity by identifying factors (model components), but also feature-wise sparsity by means of the variable weights.

For the synthetic data, a MOFA model was generated yielding 8 latent factors (model components). The weights were plot as shown in Additional file 1: Figure S2 and the explained variation was calculated. For the Hybrid Aspen data, an 8-component MOFA model was generated. Due to MOFA characteristics, 314 protein variables needed to be removed because of having nearly zero variance, and the model was built with the remaining 2818 protein variables. The absolute loadings were plotted, and the explained variation of the model calculated.

\section{Variable importance assessment using block-sPLS}

Block-sPLS [31,32] is a one-step method that combines data integration and variable selection by using partial least squares (PLS). Sparsity is achieved by applying a LASSO penalization of the PLS loading vectors when computing a singular value decomposition. The Q2 parameter is used to select the number of model components, and the root mean square error of prediction serves as criterion to evaluate the predictive power of the variables between the original (non-penalized) PLS model and the sparse PLS model. Therefore, the resulting selected variables are appropriate for prediction purposes.

In order to compare the feature selection results of MB-VIOP and block-sPLS, three 6-component block-sPLS models were generated using different constraint degrees for the Hybrid Aspen data (see Table 5). Both canonical and regression modes were tested, leading to better results when the canonical approach was used. The model was built using the canonical mode available from the mixOmics R-package that is appropriate to ensure that all data matrices are considered descriptors in a symmetric framework similar to the one used in MB-VIOP. A design matrix was set to maximize correlations among the data blocks. The resulting selected variables and the percentage of total explained variation were compared to MB-VIOP.

\section{Materials and software}

The code of the MB-VIOP algorithm was developed using MATLAB version R2019b (The MathWorks Inc., Natick, MA, USA). The four-block synthetic data set (SD16_235GLU), the block-scaling preprocesses, the OnPLS models, and the MB-VIOP results (values and plots) were also done using MATLAB (The MathWorks Inc., Natick, MA, USA). The Marzipan dataset [53] was provided by the University of Copenhagen through the website www. models.life.ku.dk/Marzipan, and preprocessed using PLS-toolbox version 8.1.1 (Eigenvector Research, Inc.). The block-sPLS analysis was performed using the mixOmics R-package version 6.8.5. The MOFA analysis was performed using the MOFA R-package version 1.6.1.

\section{Synthetic dataset (four blocks)}

The synthetic dataset, named SD16_235GLU, was created by the authors for testing and validating the MB-VIOP MATLAB code. The name of the dataset, SD16_235GLU, stands for synthetic data ( $\underline{\mathrm{SD}})$ designed in $20 \underline{16}$ for having $\underline{2}$ global components $(\underline{\mathrm{G}}), \underline{3}$ local components $(\underline{\mathrm{L}})$, and $\underline{5}$ unique components $(\underline{\mathrm{U}})$. The dataset is conformed of four data blocks $\left(\mathbf{D}_{1}, \mathbf{D}_{2}, \mathbf{D}_{3}, \mathbf{D}_{4}\right)$ and 50 observations (samples) common to all blocks. The first block $\left(\mathbf{D}_{\mathbf{1}}\right)$ contains 61 manifest variables, the second block $\left(\mathbf{D}_{2}\right)$ contains 79 , and the third and 
fourth blocks ( $\mathbf{D}_{3}$ and $\left.\mathbf{D}_{4}\right)$ contain 96 manifest variables each one. The joint (predictive) normalized loadings $\left(\mathbf{p}_{\mathbf{g}}, \mathbf{p}_{\mathbf{l}}\right)$ were created using Gaussian pure profiles, which are visualized as a bell shape in the plots; whereas the unique (orthogonal) normalized loadings $\left(\mathbf{p}_{\mathbf{u}}\right)$ were created using unit pulse pure profiles, visualized as a rectangular step in the plots. The scores, both predictive $\left(\mathbf{t}_{\mathbf{g}}, \mathbf{t}_{\mathbf{l}}\right)$ and orthogonal $\left(\mathbf{t}_{\mathbf{u}}\right)$, were randomly generated, mean-centered, scaled to unit norm, and orthogonalized among themselves. The latent variables (components) were calculated as the individual products of scores and transposed normalized loadings $\left(\mathbf{t}_{a} \mathbf{p}_{a}{ }^{\mathrm{T}}\right)$. Finally, the four data blocks were created as the sum of global, local and unique components plus the residual matrices $\mathbf{R}$. The noise was randomized, and its level was set to $0.1 \%$. A generic D-block is described in Eq. 5; where $A_{g}$ stands for the total number of global components, $A_{l}$ represents the total number of local components, and $A_{u}$ the total number of unique components. All blocks follow the pattern of Eq. 5.

$$
\mathbf{D}=\sum_{\mathrm{ag}_{\mathrm{g}}}^{\mathrm{A}_{\mathrm{g}}} \mathbf{t}_{\mathbf{a}_{\mathbf{g}}} \mathbf{p}_{\mathbf{a}_{\mathrm{g}}}^{\mathbf{T}}+\sum_{\mathrm{a}_{\mathrm{l}}}^{\mathrm{A}_{\mathrm{l}}} \mathbf{t}_{\mathbf{a}_{\mathbf{l}}} \mathbf{p}_{\mathbf{a}_{\mathbf{l}}}^{\mathbf{T}}+\sum_{\mathrm{a}_{\mathrm{u}}}^{\mathrm{A}_{\mathrm{u}}} \mathbf{t}_{\mathbf{a}_{\mathbf{u}}} \mathbf{p}_{\mathbf{a}_{\mathbf{u}}}^{\mathbf{T}}+\mathbf{R}
$$

Equations 6 - 9 show the combination of components for each data matrix. To simulate a global component, the corresponding score vector $\left(\mathbf{t}_{\mathbf{a g}}\right)$ was shared among all blocks; for the local components, the corresponding score vector $\left(\mathbf{t}_{\mathbf{a l}}\right)$ was shared among the locally connected blocks for that specific local component; and for the unique components individual scores $\left(\mathbf{t}_{\mathbf{a u}}\right)$ were used.

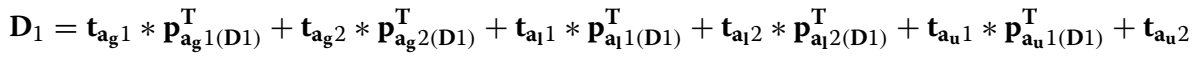

$$
\begin{aligned}
& * \mathbf{p}_{\mathbf{a}_{\mathbf{u}} 2(\mathrm{D} 1)}^{\mathbf{T}}+\mathbf{t}_{\mathbf{a}_{\mathbf{u}} 3} * \mathbf{p}_{\mathbf{a}_{\mathbf{u}} 3(\mathrm{D} 1)}^{\mathbf{T}}+\mathbf{R}_{1} \\
& \mathbf{D}_{2}=\mathbf{t}_{\mathbf{a g}_{\mathbf{g}} 1} * \mathbf{p}_{\mathbf{a}_{\mathbf{g}} 1(\mathbf{D} 2)}^{\mathbf{T}}+\mathbf{t}_{\mathbf{a}_{\mathbf{g}} 2} * \mathbf{p}_{\mathbf{a}_{\mathbf{g}} 2(\mathrm{D} 2)}^{\mathbf{T}}+\mathbf{t}_{\mathbf{a}_{\mathbf{1}} 2} \mathbf{p}_{\mathbf{a}_{\mathbf{1}} 2(\mathbf{D} 2)}^{\mathbf{T}}+\mathbf{t}_{\mathbf{a}_{1} 3} * \mathbf{p}_{\mathbf{a}_{1} 3(\mathbf{D} 2)}^{\mathbf{T}}+\mathbf{R}_{2} \\
& \mathbf{D}_{3}=\mathbf{t}_{\mathbf{a g}_{\mathrm{g}}} * \mathbf{p}_{\mathbf{a g}_{\mathbf{g}} 1(\mathbf{D} 3)}^{\mathbf{T}}+\mathbf{t}_{\mathbf{a g}_{\mathbf{g}} 2} * \mathbf{p}_{\mathbf{a g}_{2}(\mathbf{D} 3)}^{\mathbf{T}}+\mathbf{t}_{\mathbf{a}_{1} 3} * \mathbf{p}_{\mathbf{a}_{1} 3(\mathbf{D} 3)}^{\mathbf{T}}+\mathbf{t}_{\mathbf{a}_{\mathbf{u}} 4} * \mathbf{p}_{\mathbf{a}_{\mathbf{u}} 4(\mathbf{D} 3)}^{\mathbf{T}}+\mathbf{R}_{3} \\
& \mathbf{D}_{4}=\mathbf{t}_{\mathbf{a g}_{\mathbf{g}} 1} * \mathbf{p}_{\mathbf{a}_{\mathbf{g}} 1(\mathbf{D} 4)}^{\mathbf{T}}+\mathbf{t}_{\mathbf{a}_{\mathbf{g}} 2} * \mathbf{p}_{\mathbf{a}_{\mathbf{g}} 2(\mathrm{D} 4)}^{\mathbf{T}}+\mathbf{t}_{\mathbf{a}_{1} 1} * \mathbf{p}_{\mathbf{a}_{1} 1(\mathbf{D} 4)}^{\mathbf{T}}+\mathbf{t}_{\mathbf{a}_{1} 3} * \mathbf{p}_{\mathbf{a}_{1} 3(\mathbf{D} 4)}^{\mathbf{T}}+\mathbf{t}_{\mathbf{a}_{\mathbf{u}} 5} * \mathbf{p}_{\mathbf{a}_{\mathbf{u}} 5(\mathbf{D} 4)}^{\mathbf{T}}+\mathbf{R}_{4}
\end{aligned}
$$

The SD16_235GLU was designed (i) to be exigent/difficult in relation to the five unique components when modelling, (ii) to have one local component shared by three data blocks $\left(\mathbf{D}_{2}, \mathbf{D}_{3}, \mathbf{D}_{4}\right)$, (iii) to have a local component shared by $\mathbf{D}_{1}$ and $\mathbf{D}_{4}$, (iv) to have a local component shared by $\mathbf{D}_{\mathbf{1}}$ and $\mathbf{D}_{2}$, and (v) to have two global components shared by all data blocks. The percentage of variation per component is: $14.3 \%$ in $\mathbf{D}_{1}, 25 \%$ in $\mathbf{D}_{2}$, $25 \%$ in $\mathbf{D}_{3}$, and $20 \%$ in $\mathbf{D}_{4}$ (thus, $\mathbf{D}_{\mathbf{1}}$ has a total of seven components, $\mathbf{D}_{\mathbf{2}}$ has four, $\mathbf{D}_{\mathbf{3}}$ also four, and $\mathbf{D}_{4}$ has five).

\section{Marzipan dataset (six blocks).}

The Marzipan dataset consists of six data blocks obtained from the analysis of thirty-two marzipan samples, of nine different recipes, performed using six different spectrometers set-ups. The marzipan samples contained different amounts of almonds, apricot kernels, 
water, sucrose, invert sugar, glucose syrup, and minor contributions of additives; cocoa was added in some of the marzipan samples, giving them a distinctive brown color. The six spectrometers (including optical principles, spectral range, and other details) were described by Christensen et al. [53] in 2004. An additional set of measurements using an InfraAlyzer 260 spectrometer was originally considered as a seventh data block [53], but it has been excluded from this work because of not using exactly the same samples than the other six instrumental analyses.

The first data block (NIRS1) contained 1000 variables $(400-2500 \mathrm{~nm})$, and the second data block (NIRS2) had 600 variables $(800-2100 \mathrm{~nm}$ ); both NIRS1 and NIRS2 datasets were obtained using a NIRSystems 6500 spectrometer. The third (from an Infraprover II instrument) contained 406 variables, the fourth (from a Bomem MB 160 Diffusir) consisted of 664 variables, the fifth (from an Infratec 1255) had 100 variables, and the sixth (from a PerkinElmer System 2000) had 950 variables. Thus, the dimensions of the different data blocks varied from 100 to 1000 variables (i.e., a ten times difference between the smallest and the largest). NIRS1, Infraprover II and Bomem data blocks were preprocessed by extended multiplicative signal correction (EMSC) [54]; whilst NIRS2, Infratec and PerkinElmer data blocks were preprocessed by Savitsky-Golay differentiation (2nd derivative, 3rd order, 15 points window) [55]. In addition, all data blocks were meancentered and normalized to equal sum of squares before building the OnPLS model.

\section{Metabolomics, proteomics and transcriptomics data of hybrid aspen (three blocks)}

The Hybrid Aspen dataset used here, previously pretreated and analyzed in Bylesjö et al. [56] in 2009 and in Löfstedt et al. [57] in 2013, contains thirty-three samples of hybrid aspen (Populus tremula $x$ Populus tremuloides) labeled according to the plant internode from where they were sampled (categories A, B, and C) and according to three different genotypes of hybrid aspen (WT, G5, and G3). The wild type (WT) played the role of reference sample. The G5 and G3 genotypes were related to the PttMYB21a gene, which is known to primarily affect lignin biosynthesis and plant growth characteristics. The G5 genotype contained several antisense constructs of the PttMYB21a gene, affecting plant growth; thus, this genotype displays a distinct phenotype with slower growth compare to the WT samples. The G3 genotype contained only one antisense construct of the PttMYB21 a gene, displaying a similar but less distinct phenotype compared to the G5 samples. Further details are described by Bylesjö et al. [56].

All thirty-three samples were measured for transcript (cDNA), protein (UPLC/MS) and metabolite (GC/TOFMS) quantities [57]. As result, three data blocks were obtained: a transcript data block containing 14,738 variables (microarray elements), a protein data block containing 3132 variables (extracted chromatographic peaks), and a metabolite data block containing 281 variables (extracted chromatographic peaks). 


\section{Abbreviations}

block-sPLS: Multiblock sparse partial least squares; CPCA: Consensus principal component analysis; GSVD: Generalized singular value decomposition; HPCA: Hierarchical principal component analysis; HPLS: Hierarchical partial least squares; JIVE: Joint and individual variation explained; MBPLS: Multiblock partial least squares; MB-VIOP: Multiblock variable influence on orthogonal projections; MOFA: Multi-omics factor analysis; msPLS: Multiset sparse partial least squares; OPLS: Orthogonal projections to latent structures; O2PLS: 2-Block orthogonal projections to latent structures; OnPLS: $\mathrm{N}$-block orthogonal projections to latent structures; PCA: Principal component analysis; PLS: Partial least squares to latent structures; RGCCA: Regularized generalized canonical correlation analysis; RMSEP: Root mean square error of prediction; SGCCA: Sparse generalized canonical correlation analysis; sPLS: Sparse partial least squares; SSX: Sum of squares of X; SSY: Sum of squares of Y; VIP: Variable influence on projection.

\section{Supplementary Information}

The online version contains supplementary material available at https://doi.org/10.1186/s12859-021-04015-9.

Additional file 1: Supporting information that contains Tables S1-S5 and Figures S1-S5.

\section{Acknowledgements}

The authors want to thank the anonymous reviewers for helping to improve this paper, the Chemistry Department of Umeå University where MB-VIOP was developed as part of the PhD thesis of BGP, and the University of Copenhagen for providing the Marzipan dataset via the website www.models.life.ku.dk/Marzipan.

\section{Authors' contributions}

For the MB-VIOP algorithm, BGP generated the theory, equations, MATLAB code, results and figures for the three datasets during her PhD under the supervision of JT and PG. BGP also generated the R codes and results for the comparisons using the MOFA and the block-sPLS methods. JT provided the OnPLS models generated using the OnPLS algorithm/ code. PG advised on the theory and equations of MB-VIOP, method validation and spectroscopy interpretation. BGP wrote the manuscript draft, and PG checked it and improved it. The manuscript was revised and approved by all authors. All authors read and approved the final manuscript.

\section{Funding}

The authors are grateful for the financial support given by MKS Instruments AB (BGP), ESSENCE (JT), and Industrial Doctoral School (BGP), Umeå University, Sweden. In addition, part of this work was carried out during the tenure of an ERCIM "Alain Bensoussan"Fellowship Programme (BGP). The funding body did not play any roles in the design of the study and collection, the analysis, the data interpretation, or the manuscript writing.

\section{Availability of data and materials}

The Marzipan dataset analyzed in the current study is available through the website www.models.life.ku.dk/Marzipan of the University of Copenhagen. The Hybrid Aspen and the SD16_235GLU datasets are available from the authors on reasonable request.

\section{Ethics approval and consent to participate}

Not applicable.

\section{Consent for publication}

Not applicable.

\section{Competing interests}

The authors declare that they have no competing interests.

\section{Author details}

${ }^{1}$ Department of Chemistry, Computational Life Science Cluster (CLiC), Umeå University, Umeå, Sweden. ${ }^{2}$ Industrial Doctoral School (IDS), Umeå, Sweden. ${ }^{3}$ Department of Engineering Cybernetics (ITK), Norwegian University of Science and Technology (NTNU), Trondheim, Norway. ${ }^{4}$ Helen and Robert Appel Alzheimer's Disease Research Institute, Feil Family Brain and Mind Research Institute, Weill Cornell Medicine (WCM), Cornell University, New York, NY, USA. ${ }^{5}$ Forest Biomaterials and Technology, Swedish University of Agricultural Sciences (SLU), Umeå, Sweden. ${ }^{6}$ Sartorius Corporate Research, Umeå, Sweden.

Received: 16 July 2020 Accepted: 10 February 2021

Published online: 03 April 2021 


\section{References}

1. Horst P. Relations among $m$ sets of measures. Psychometrika. 1961;26:129-49.

2. Levin J. Simultaneous factor analysis of several Gramian matrices. Psychometrika. 1966;31:413-9.

3. Curran PJ, Hussong AM. Integrative data analysis: The simultaneous analysis of multiple data sets. Psychol Methods. 2009;14:81-100

4. Kourti T, Nomikos P, MacGregor JF. Analysis, monitoring and fault diagnosis of batch processes using multiblock and multiway PLS. J Process Control. 1995:5:277-84.

5. Westerhuis JA, Kourti T, MacGregor JF. Analysis of multiblock and hierarchical PCA and PLS models. J Chemom. 1998;12:301-21.

6. Frank I, Feikema J, Constantine N, Kowalski B. Prediction of product quality from spectral data using the partial leastsquares method. J Chem Inf Comput Sci. 1984:24:20-4.

7. Mazerolles G, Boccard J, Hanafi M, Rudaz S. Analysis of experimental design with multivariate response: a contribution using multiblock techniques. Chemom Intell Lab Syst. 2011;106:65-72.

8. Conesa A, Prats-Montalbán JM, Tarazona S, Nueda MJ, Ferrer A. A multiway approach to data integration in systems biology based on Tucker3 and N-PLS. Chemom Intell Lab Syst. 2010;104:101-11.

9. Reinke SN, Galindo-Prieto B, Skotare T, Broadhurst DI, Singhania A, Horowitz D, Djukanović R, Hinks TSC, Geladi P, Trygg J, Wheelock CE. OnPLS-based multi-block data integration: a multivariate approach to interrogating biological interactions in asthma. Anal Chem. 2018:90:13400-8.

10. Qannari EM, Wakeling I, Courcoux P, MacFie HJH. Defining the underlying sensory dimensions. Food Qual Prefer. 2000;11:151-4.

11. Tenenhaus M, Pagès J, Ambroisine L, Guinot C. PLS methodology to study relationships between hedonic judgements and product characteristics. Food Qual Prefer. 2005;16:315-25.

12. Geladi P, Kowalski BR. Partial least-squares regression: a tutorial. Anal Chim Acta. 1986;185:1-17.

13. Wold S, Martens $\mathrm{H}$, Wold $\mathrm{H}$. The multivariate calibration-problem in chemistry solved by the PLS method. Lecture Notes Math. 1983;973:286-93.

14. Wold, S., Hellberg, S., Lundstedt, T., Sjöström, M. \& Wold, H. PLS modeling with latent variables in two or more dimensions. in Symposium on PLS model building: theory and application. (1987).

15. Geladi, P., Martens, H., Martens, M., Kalvenes, S. \& Esbensen, K. Multivariate comparison of laboratory measurements. in Proc. Symposium on Applied Statistics 49-61 (1988).

16. Wold S, Kettaneh N, Tjessem K. Hierarchical multiblock PLS and PC models for easier model interpretation and as an alternative to variable selection. J Chemom. 1996;10:463-82.

17. Wangen LE, Kowalski BR. A multiblock partial least squares algorithm for investigating complex chemical systems. J Chemom. 1988;3:3-20.

18. Wise BM, Gallagher NB. The process chemometrics approach to process monitoring and fault detection. J Process Control. 1996;6:329-48.

19. Tenenhaus A, Tenenhaus M. Regularized generalized canonical correlation analysis. Psychometrika. 2011;76:257-84.

20. Qin SJ, Valle S, Piovoso MJ. On unifying multiblock analysis with application to decentralized process monitoring. J Chemom. 2001;15:715-42

21. el Bouhaddani S, Uh HW, Jongbloed G, Hayward C, Klarić L, Kiełbasa SM, Houwing-Duistermaat J. Integrating omics datasets with the OmicsPLS package. BMC Bioinform. 2018;19:371.

22. Trygg J. O2-PLS for qualitative and quantitative analysis in multivariate calibration. J Chemom. 2002;16:283-93.

23. Smilde AK, Westerhuis JA, de Jong S. A framework for sequential multiblock component methods. J Chemom. 2003; 17:323-37.

24. Gabrielsson J, Jonsson H, Airiau C, Schmidt B, Escott R, Trygg J. The OPLS methodology for analysis of multi-block batch process data. J Chemom. 2006;20:362-9.

25. Höskuldsson A. Multi-block and path modelling procedures. J Chemom. 2008;22:571-9.

26. Hanafi M, Kohler A, Qannari EM. Shedding new light on hierarchical principal component analysis. J Chemom. 2010;24:703-9.

27. Mazerolles G, Preys S, Bouchut C, Meudec E, Fulcrand H, Souquet JM, Cheynier V. Combination of several mass spectrometry ionization modes: a multiblock analysis for a rapid characterization of the red wine polyphenolic composition. Anal Chim Acta. 2010;678:195-202.

28. El Ghaziri A, Cariou V, Rutledge DN, Qannari EM. Analysis of multiblock datasets using ComDim: overview and extension to the analysis of $(K+1)$ datasets. J Chemom. 2016;30:420-9.

29. Jourdren S, Saint-Eve A, Panouillé M, Lejeune P, Déléris I, Souchon I. Respective impact of bread structure and oral processing on dynamic texture perceptions through statistical multiblock analysis. Food Res Int. 2016;87:142-51.

30. Smilde, A., Bro, R. \& Geladi, P. Multi-way analysis: applications in the chemical sciences. in 1-18221-349 (John Wiley and Sons, 2004)

31. Lê Cao KA, Rossouw D, Robert-Granié C, Besse P. A sparse PLS for variable selection when integrating omics data. Stat Appl Genet Mol Biol. 2008;7:35.

32. Rohart, F., Gautier, B., Singh, A. \& Lê Cao, K.-A. mixOmics: An R package for 'omics feature selection and multiple data integration. PLOS Computational Biology 13, e1005752 (2017).

33. Argelaguet, R., Velten, B., Arnol, D., Dietrich, S., Zenz, T., Marioni, J. C., Buettner, F., Huber, W. \& Stegle, O. Multi-omics factor analysis - a framework for unsupervised integration of multi-omics data sets. Mol Syst Biol. 14, (2018).

34. Löfstedt T, Trygg J. OnPLS-a novel multiblock method for the modelling of predictive and orthogonal variation. J Chemom. 2011;25:441-55.

35. Lock EF, Hoadley KA, Marron JS, Nobel AB. Joint and individual variation explained (JIVE) for integrated analysis of multiple data types. Ann Appl Stat. 2013;7:523-42.

36. Van Loan CF. Generalizing the Singular Value Decomposition. SIAM J Numer Anal. 1976;13:76-83.

37. Csala A, Zwinderman AH, Hof MH. Multiset sparse partial least squares path modeling for high dimensional omics data analysis. BMC Bioinform. 2020;21:9.

38. Andersen CM, Bro R. Variable selection in regression-a tutorial. J Chemom. 2010;24:728-37. 
39. Galindo-Prieto B, Eriksson L, Trygg J. Variable influence on projection (VIP) for orthogonal projections to latent structures (OPLS). J Chemom. 2014;28:623-32.

40. Kvalheim OM, Arneberg R, Bleie O, Rajalahti T, Smilde AK, Westerhuis JA. Variable importance in latent variable regression models. J Chemom. 2014;28:615-22.

41. Leardi R. Genetic algorithms in chemometrics and chemistry: a review. J Chemom. 2001;15:559-69.

42. Lindgren, F., Geladi, P., Rännar, S. \& Wold, S. Interactive variable selection (IVS) for PLS. Part 1: Theory and algorithms. $J$ Chemom. 8, 349-363 (1994).

43. Lindgren, F., Geladi, P., Berglund, A., Sjöström, M. \& Wold, S. Interactive variable selection (IVS) for PLS. Part II: Chemical applications. J. Chemom. 9, 331-342 (1995).

44. Galindo-Prieto B, Eriksson L, Trygg J. Variable influence on projection (VIP) for OPLS models and its applicability in multivariate time series analysis. Chemom Intell Lab Syst. 2015:146:297-304.

45. Farrokhnia M, Karimi S. Variable selection in multivariate calibration based on clustering of variable concept. Anal Chim Acta. 2016;902:70-81.

46. Nørgaard L, Saudland A, Wagner J, Nielsen JP, Munck L, Engelsen SB. Interval partial least-squares regression (iPLS): A comparative chemometric study with an example from near-infrared spectroscopy. Appl Spectrosc. 2000;54:413-9.

47. Tibshirani, R. Regression Shrinkage and Selection via the Lasso. J R Stat Soc. Ser B (Methodological) 58, 267-288 (1996).

48. Galindo-Prieto B, Trygg J, Geladi P. A new approach for variable influence on projection (VIP) in O2PLS models. Chemom Intell Lab Syst . 2017;160:110-24.

49. Tenenhaus A, Philippe C, Guillemot V, Le Cao KA, Grill J, Frouin V. Variable selection for generalized canonical correlation analysis. Biostatistics. 2014;15:569-83.

50. Wold, S., Johansson, E. \& Cocchi, M. PLS - partial least-squares projections to latent structures. 3D QSAR Drug Design (Ed. Kubinyi H.), Theory Methods and Applications, ESCOM Science Publishers, Leiden 523-550 (1993).

51. Galindo-Prieto, B. Novel variable influence on projection (VIP) methods in OPLS, O2PLS, and OnPLS models for single-and multi-block variable selection: VIPOPLS, VIPO2PLS, and MB-VIOP methods. (Umeå University, 2017).

52. Sunoj S, Igathinathane C, Visvanathan R. Nondestructive determination of cocoa bean quality using FT-NIR spectroscopy. Comput Electron Agric. 2016;124:234-42.

53. Christensen J, Nørgaard L, Heimdal H, Pedersen J, Engelsen S. Rapid spectroscopic analysis of marzipan —comparative instrumentation. J Near Infrared Spectrosc. 2004;12:63-75.

54. Martens H, Stark E. Extended multiplicative signal correction and spectral interference subtraction: new preprocessing methods for near infrared spectroscopy. J Pharm Biomed Anal. 1991;9:625-35.

55. Savitzky A, Golay MJE. Smoothing and differentiation of data by simplified least squares procedures. Anal Chem. 1964;36:1627-39.

56. Bylesjö M, Nilsson R, Srivastava V, Grönlund A, Johansson Al, Jansson S, Karlsson J, Moritz T, Wingsle G, Trygg J. Integrated analysis of transcript, protein and metabolite data to study lignin biosynthesis in hybrid aspen. J Proteome Res. 2009;8:199-210.

57. Löfstedt T, Hoffman D, Trygg J. Global, local and unique decompositions in OnPLS for multiblock data analysis. Anal Chim Acta. 2013;791:13-24.

\section{Publisher's Note}

Springer Nature remains neutral with regard to jurisdictional claims in published maps and institutional affiliations.

Ready to submit your research? Choose BMC and benefit from:

- fast, convenient online submission

- thorough peer review by experienced researchers in your field

- rapid publication on acceptance

- support for research data, including large and complex data types

- gold Open Access which fosters wider collaboration and increased citations

- maximum visibility for your research: over 100M website views per year

At BMC, research is always in progress.

Learn more biomedcentral.com/submissions 